\title{
Detection and Classification of Adult and Fetal ECG Using Recurrent Neural Networks, Embedded Volterra and Higher-Order Statistics
}

\author{
Walid A. Zgallai1,2 \\ ${ }^{1}$ Wokingham \\ ${ }^{2} \mathrm{Dubai}$ \\ ${ }^{1} U . K$. \\ 2U. A. E.
}

\section{Introduction}

The fetal heart rate (FHR) is a useful tool in the assessment of the condition of the fetal before and during labour. Fetal Electrocardiography (FECG) (Sureau, 1996) uses noninvasive surface electrodes placed on the maternal abdomen is another tool for FHR recording (Sureau, 1996). The fetal signal is weak relative to the maternal signal and to the competing noise. Widrow et al. (Widrow et al., 1975) proposed an adaptive filtering and adaptive noise cancellation method to extract the FECG from the composite maternal ECG signal. Auto-correlation and cross correlation techniques (Van Bemmel, 1968) and spatial filtering techniques (Van Oosterom, 1986, and Bergveld and Meijier 1981) have been proposed. These methods require multiple maternal thoracic ECG signals. Other methods were proposed for the rejection of the disturbing maternal ECG signal (Sureau, 1996). The automated long-term evaluation of FECG is regarded as less robust than CTG. A failure rate of approximately $30 \%$ is quoted as an almost unanimous norm (Herbert et al., 1993). The advantage of FECG is that it can be implemented in small and relatively low-cost devices (Lin et al., 1997).

A proposed technique employing wavelet transform (Khamene and Negahadariapour, 2002) exploits the most distinct features of the signal, leading to more robustness with respect to signal perturbations. The algorithm is validated using high SNR data. Dynamic modelling has been proposed (Schreiber, and D Kaplan, 1996). The data has comparatively high SNR and the fetal heartbeats can be detected by an adaptive matched filter and requires much shorter data samples than the dynamic modelling. The dynamic modelling apparent success at high SNR is offset by the required lengthy data. Due to the beat-to-beat fluctuations of the shape and duration of the ECG waveform, the normal ECG cannot be considered to be deterministic. Determinism is found in adult and fetal ECGs for data lengths of 10,000 samples (Rizk et al., 2002). The independent component analysis (ICA) has been carried out under assumptions (Lathauwer et al., 2000), the validity of each has been challenged (Rizk et al., 2001). 
This chapter investigates the application of recurrent neural networks to the detection and classification of ECG signals. Third-order cumulants, bispectrum, polyphase, and embedded Volterra are utilised. The chapter develops methodology for adult ECG detection using the higher-order statistics. It extends that to non-invasive fetal heartbeat detection, during labour. The work is also extended to classify adult heart abnormalities based on the phases of the higher-order statistics. The chapter is organised as follows; Sections 2 and 3 employ third-order cumulant slices and bispectrum contours, respectively, to detect adult and fetal ECG signals. Section 4 introduces a method of ECG abnormality detection using polyphase. Section 5 shows how third-order cumulants could be utilised for the detection of late potentials. Section 6 summarises the conclusions.

\section{ECG third-order cumulant slices classification}

\subsection{Background}

Many techniques have been introduced to detect fetal heartbeats during labour. The advances in higher-order statistics, non-linear filtering, and artificial neural networks are exploited to propose a hybrid technique to improve the non-invasive detection of fetal heartbeats during labour. A third-order cumulants (TOCs) technique for non-invasive fetal heartbeat detection has been proposed (Zgallai et al., 1997). The ECG signal is processed using a Volterra filter. To improve the performance of the Volterra filter, quadratic and cubic LMF Volterra filters have been proposed (Zgallai et al., 2007). The proposed system uses the mother and fetal third-order cumulants (TOCs), which carry the signature imprints of their respective QRS-complexes, in the signal processing phase.

Quadratic and cubic Volterra filters with LMF updates have been employed to synthesise the signal into linear, quadratic, and cubic parts, and retain only the linear part. The classification phase employs an LMS-based single-hidden-layer perceptron. This section proposes incorporating an adaptive cubic LMF Volterra and an artificial neural network classifier to improve the detection rate (Zgallai, 2010). The cubic Volterra filter has been shown to improve the performance of some biomedical signals such as electromyographic signals during labour (Zgallai et al., 2009). Cross validation has been done by comparing the results of the detection to QRS peaks of the fetal heartbeat extracted from the fetal scalp electrode which is the goldstandard.

\subsection{Third-order cumulants}

A non-Gaussian signal $\{X(k)\}$ has TOCs given by (Nikias and Petropulu, 1993):

$$
\mathrm{C}_{3}^{\mathrm{x}}\left(\tau_{1}, \tau_{2}\right)=\operatorname{Cum}\left\{\mathrm{X}(\mathrm{k}), \mathrm{X}\left(\mathrm{k}+\tau_{1}\right), \mathrm{X}\left(\mathrm{k}+\tau_{2}\right)\right\} .
$$

The calculations of the TOCs are implemented off-line due to the large CPU time required to calculate the lags. One way of reducing this load is to use 1-d slices of the TOCs. Onedimensional slices of $\mathbf{C}_{3}{ }^{\mathrm{x}}\left(\tau_{1}, \tau_{2}\right)$ can be defined as (Nikias and Petropulu, 1993):

$$
\mathrm{r}_{2,1}^{\mathrm{x}}(\tau) \underline{=} \operatorname{Cum}\{\mathrm{X}(\mathrm{k}), \mathrm{X}(\mathrm{k}), \mathrm{X}(\mathrm{k}+\tau)\}=\mathrm{c}_{3}^{\mathrm{x}}(0, \tau)
$$




$$
\mathrm{r}_{1,2}^{\mathrm{x}}(\tau) \underline{\underline{\nabla}} \operatorname{Cum}\{\mathrm{X}(\mathrm{k}), \mathrm{X}(\mathrm{k}+\tau), \mathrm{X}(\mathrm{k}+\tau)\}=\mathrm{C}_{3}^{\mathrm{x}}(\tau, \tau)
$$

where $r_{2,1}(\tau)$ and $r_{1,2}(\tau)$ represent 1-d wall and diagonal slices, respectively. The former can be obtained from Eq. (2.1) by assuming $\tau_{1}=0$. The later obeys the condition $\tau_{1}=\tau_{2}$. Employing 1-d slices will have the effect of reducing the CPU time by reducing the complexity of the operations. The calculations of TOC slices are comparable to those of autocorrelation and take CPU time of approximately 1 msec unlike TOCs, which take 1 sec to calculate. For a sampling rate of $0.5 \mathrm{KHz}$ and an FHR of the order of $120 \mathrm{bpm}$, a real-time system can be implemented. An algorithm which calculates any arbitrarily chosen off diagonal and off wall one-dimensional slice, and hence reduce the CPU time by $99 \%$, has been developed (Zgallai, 2007).

Adequate knowledge of the TOC of both the maternal and fetal ECG signals must first be acquired in order to pave the way for fetal QRS-complex identification and detection. There are several motivations behind using TOC in processing ECG signals; (i) ECG signals are predominantly non-Gaussian (Rizk and Zgallai, 1999), and exhibit quadratic and higherorder non-linearities supported by third- and fourth-order statistics, respectively. (ii) Gaussian noise diminishes in the TOC domains if the data length is adequate (Nikias and Petropulu, 1993). It is possible to process the ECG signal in Gaussian noise-free domains. For ECG signals a minimum length of $1 \mathrm{sec}$ is adequately long to suppress Gaussian noise in the TOC domains, whilst not long enough to violate Hinich's criterion of local stationarity (Brockett et al., 1988). In general, ECG signals are non-stationary in the statistical sense, but relatively short data can be successfully treated with conventional signal processing tools primarily designed for stationary signals. When dealing with individual cardiac cycles, nonstationarity is not an issue but when one takes on board the heart rate time series which is chaotic and multi-dimensional then it is not wise to assume stationarity for analysis purposes. (iii) In the TOC domain all sources of noise with symmetric probability density functions (pdfs), e.g., Gaussian and uniform, will vanish. The ECG signals are retained because they have non-symmetric distributions (Zgallai et al., 1997). (iv) ECG signals contain measurable quantities of quadratic and, to a lesser extent, cubic non-linearities. Such measurable quantities of non-linearity, if not synthesised and removed before any further processing for the purpose of signal identification and classification, could lead to poor performance with regard to fetal QRS-complex detection rates.

\subsection{LMF quadratic and cubic volterra}

The Volterra structure is a series of polynomial terms (Schetzen, 1980) which are formed from a time sequence $\{\mathrm{y}(\mathrm{k})$. The output of the filter is expressed as

$$
\mathrm{y}(\mathrm{n})=\sum_{\mathrm{i}_{1}=1}^{\mathrm{N}} \mathrm{a}_{\mathrm{i}_{1}}^{1} \mathrm{x}_{\mathrm{k}-\mathrm{i}_{1}+1}+\sum_{\mathrm{i}_{1}=1}^{\mathrm{N}} \sum_{\mathrm{i}_{2}=1}^{\mathrm{N}} \mathrm{a}_{\mathrm{i}_{1}, \mathrm{i}_{2}}^{2} \mathrm{x}_{\mathrm{k}-\mathrm{i}_{1}+1} \mathrm{x}_{\mathrm{k}-\mathrm{i}_{2}+1}+\ldots
$$

Adaptive conventional Volterra is updated using the Least-Mean Squares (LMS) criterion. The LMS algorithm minimises the expected value of the squared difference between the estimated output and the desired response. A more general case is to minimise $E\left\{e(n)^{2 N}\right\}$ (Wallach and Widrow, 1984). $\mathrm{N}=2$ is the Least-Mean-Fourth (LMF). The LMF algorithm updates the weights as follows: 


$$
\mathbf{a}_{i}(n+1)=\mathbf{a}_{i}(n)+2 \mu_{i} \cdot e^{3}(n) \cdot x(n) .
$$

The LMF has a faster convergence than the LMS algorithm. It has generally a lower weight noise than the LMS algorithm, with the same speed of convergence. It was shown to have 3 $\mathrm{dB}$ to $10 \mathrm{~dB}$ lower mean-squared error (MSE) than the LMS algorithm (Wallach and Widrow, 1984). Adaptive LMF-based quadratic and cubic Volterra structures have been developed and shown to outperform LMS-based Volterra by 6-7 dBs (Zgallai, 2007).

\subsection{Neural network classifiers}

A major limitation of the back-propagation algorithms is the slow rate of convergence to a global minimum of the error-performance surface because the algorithm operates entirely on the gradient of the error-performance surface with respect to the weights in the singlehidden-layer perceptron. The back-propagation learning process is accelerated by incorporating a momentum term. The use of momentum introduces a feedback loop which prevents the learning process from being stuck at a local minimum on the errorperformance surface of the single-hidden-layer perceptron. The classifier is a single-hiddenlayer perceptrion based on a modified Back-Propagation technique. The modified backpropagation algorithm has a momentum term which helps to avoid local minima. One hundred and sixty one-dimensional TOC slices have been used as templates for the desired signals in the Artificial Neural Network (ANN) classifier.

\subsection{The proposed algorithm}

\subsubsection{TOC detection}

1. Create ECG cumulant database

2. Detect the maternal QRS-complexes - Read the ECG recording sequentially, and process each of the $90 \%$ overlapping windows (length $250 \mathrm{msec}$ ) to compute the diagonal or wall slice TOC. The slice is matched to the templates until a maternal QRS-complex is detected. An auxiliary subroutine is used to pinpoint the position of the R-wave. If the second successive segment detects a maternal QRS-complex then it is discarded because it is the same complex detected in the adjacent window. The whole process of TOC template matching is repeated until the second maternal QRS-complex is detected and its R-wave is pinpointed. The maternal heart rate is accurately calculated from the knowledge of the current and previous R-wave positions.

3. Detect the fetal cardiac cycles - The search begins from the position of the detected maternal R-wave. Window overlapping, each with fetal cumulant template matching, continues until the first, second, and possibly third fetal ECG TOC diagonal and wall slice signatures have been matched to one template for each one of them. Once the slices have been matched, the window will be flagged as a detection window. If the next overlapping window detects a fetal heartbeat, it will be discarded because it is the same fetal heartbeat that has just been detected in the previous window. The number of fetal heartbeats detected within the maternal cardiac cycle is counted. The instantaneous maternal heart rate is previously known with some degree of accuracy, and the relative fetal to maternal heartbeat is also known within the maternal cardiac cycle. Hence, the averaged fetal heart rate can be calculated within each maternal cardiac cycle. Operations 2 and 3 are repeated for all individual maternal cardiac cycles. 


\subsubsection{Window minimum length and ECG segmentation}

The duration of the fetal cardiac cycle varies from $250 \mathrm{msec}$ to $500 \mathrm{msec}$ for a range of fetal heart rate between $240 \mathrm{bpm}$ and $120 \mathrm{bpm}$. The fetal QRS-complex itself occupies between 50 msec and $70 \mathrm{msec}$. The fetal heartbeat is detected in a flag window of length $250 \mathrm{msec}$. This window length serves two criteria; (i) it is the minimum length yielding an acceptable upper threshold of both the deterministic and stochastic noise types inherent in the higher-order statistics of the ECG signals encountered, and (ii) this window length allows the detection of one, two, three, or four fetal heartbeats (FHBs) within one maternal transabdominal cardiac cycle. For example, for maternal heartbeat of $60 \mathrm{bpm}$, the R-wave-to-R-wave $=1000 \mathrm{msec}$, and four segments $\times 250 \mathrm{msec}=$ one maternal cardiac cycle $=$ possible four fetal cardiac cycles.

\subsubsection{Overlapping window}

When detecting the fetal heartbeat within the maternal transabdominal cardiac cycle, $90 \%$ overlapping windows, each of $250 \mathrm{msec}$ duration, are scanned at a rate of $100 \mathrm{~Hz}$ with a sampling rate of $0.5 \mathrm{KHz}$. The overlapping percentage should be carefully chosen to compensate for the apparent loss of temporal resolution due to a lengthy window which is dictated by the maximum threshold of the variance of the TOCs. The average fetal QRScomplex duration is $60 \mathrm{msec}$. This may be encountered at the beginning, middle, or end of the flag window. Hence by using a window overlapping of $90 \%$, any fetal QRS-complex which may be missed because it starts to evolve, say, $20 \mathrm{msec}$ before the end of a window, can definitely be picked up by the next one or two overlapping windows when it completes its full duration of $60 \mathrm{msec}$ and has definitely reached its full peak (the R-wave). If this particular QRS-complex has enough strength to be picked up by two successive overlapping windows, the algorithm will count it as one FHB. It has been found that reducing the overlapping below $90 \%$ yielded missed fetal heartbeats.

\subsubsection{Averaged fetal heart rate calculation}

The instantaneous fetal heart rate is calculated by measuring the interval between two successive R-waves. This requires pinpointing accurately the R-point of the QRS-complex. Although the ECG TOC template matching technique is very effective in detecting the occurrence of the QRS-complex as a whole even when it is completely buried in noise, it cannot locate the R-wave over a window length of $250 \mathrm{msec}$ which satisfies the criterion for the variance threshold. In most transabdominal ECG recordings (85\%), the fetal QRScomplexes cannot be seen as they are completely masked by other signals and motion artefact. This obscurity accounts for the lower success rate of fetal heartbeat detection in other assessed fetal heartbeat detection techniques (Sureau, 1996). The adult heartbeats can be measured accurately and the instantaneous heart rate for adults can be calculated. Hence, by counting the number of fetal heartbeats (FHBs) that have occurred between two successive maternal R-waves, one can easily calculate the averaged FHR within the maternal cardiac cycle. On average, the maternal cardiac cycle is $1000 \mathrm{msec}$. Two maternal cardiac cycles measure 2 sec. So, detecting and displaying up to eight FHBs will take less than $2.000030 \mathrm{sec}$ which is well within the manufacturers' detection-to-display interval of $3.75 \mathrm{sec}$. 


\subsection{Data collection and results}

\subsubsection{Data collection}

Data was obtained from pregnant women at various stages of gestation (Zgallai, 2007). This was achieved, with the consent of women, using a pair of electrodes, Sonicaid 8000, a Pentium IV PC and an interface card. The software used for the attractor calculations was MATLAB 8.0. Ag-AgCl Beckman electrodes of $8 \mathrm{~cm}$ in diameter, and 25-cm spaced centres were positioned on the abdominal wall after careful preparation of the skin, which lowers the interelectrode impedance of $10 \mathrm{k} \Omega$. The electrode pair is set over the umbilicus, and lined up with the median vertical axis of the uterus. The ground electrode is located on the woman's hip. The training data involves using third-order cumulants and their slices of segments of fetal scalp electrode measurement. These are used to compare with results obtained from the transabdominal ECG recording. The testing data is the transabdominal ECG recording.

\subsubsection{Results}

\subsubsection{TOCs and their diagonal and wall slices}

An optimised third-order Volterra structure is employed to decompose the ECG signal into its linear, quadratic, and cubic parts and retain only the linear part. Fig. 2.1 depict a maternal transabdominal ECG signal with segmentation and the corresponding TOCs and their diagonal and wall slices for predominantly maternal QRS-complexes, the first fetal heartbeats with maternal contribution, QRS-free ECGs, and the second fetal heartbeats with maternal contribution. The diagonal and wall TOC slices of the maternal QRS-complexes, segment (I) in Fig. 2.1, are easily distinguished from the diagonal and wall TOC slices of segments (II), (III), and (IV). Furthermore, the diagonal and wall TOC slices of the fetal heartbeat segments, (II) and (IV) in Fig. 2.1, are distinguishable from the corresponding diagonal and wall TOC slices of the QRS-free ECG segments (III). However, in some cases, those of segments (II) and (IV) could be mistaken for QRS-free ECG segments. The peaks of the QRS-free ECG segments are much narrower and more related to motion artefact than a signal.

\subsubsection{The NN classifier}

A single-hidden-layer perceptron is used for the classification of the TOC slices of the maternal QRS-complexes, the first fetal heartbeat with maternal contribution, QRS-free ECG, and the second fetal heartbeat with maternal contribution from maternal transabdominal ECG segments. This is achieved using a standard back-propagation with momentum algorithm (Caudill and C. Butler, 1992). Each of the input and output layers have a dimension of $8 \times 8$ and the hidden layer has a dimension of $5 \times 5$. The input to the first layer is the TOCs diagonal and wall slices. The network is trained using TOC slice templates obtained from the maternal chest and fetal scalp electrode ECGs as well as previously detected and earmarked transabdominal ECG segments. The latter training sequences are templates of the diagonal and wall slices of the TOCs of four segments from maternal transabdominal full cardiac cycles. The input to the network is eight template patterns. These are the TOC diagonal and wall slices of four segments from one transabdominal cardiac cycle. For example the first pair are maternal slices, the second pair are fetal slices, the third pair are QRS-free slices, and the fourth pair are fetal slices. The network is trained over the eight patterns. The training terminates 
when the worst error in all patterns in one pass is less than 0.1 . Typically the average error will be in the range of 0.001 .

The TOC slice templates are used as input to the classifier. Each one of the 10 templates in each set is used as an input and the weights of each neuron in the classifier are optimised by changing the learning rate and the momentum constant until the error is minimised. Then the transabdominal ECG signal with $250-\mathrm{msec}$ window is used as an input to the classifier. The instantaneous weights of the input signal are compared to those of the templates which are stored in the memory. The two sets of parameters are correlated. Once a signal is classified the output will be set to 1 . The classification of the four segments involves a pattern-by-pattern updating rather than batch updating for the weight adjustments. This is more suitable to speed up the performance. Pattern-by-pattern updating tends to be orders of magnitude faster than batch updating. However, it should be noted that pattern-by-pattern updating is harder to parallelise. Fig. 2.2 summarises the results of the optimisation process.

Parameters of the single-hidden layer perceptron: The network has been optimised in terms of its learning rate, momentum constant, and hidden layer size to achieve the minimum meansquared error. The optimum learning rate is found to be 0.8 . The optimum momentum constant is found to be 0.99 and 0.90 for the maternal QRS-complex and the fetal heartbeat with maternal contribution segments, respectively. The single-hidden-layer has an optimum dimension of $5 \mathrm{x}$ 5. The input to the first layer is the TOCs diagonal and wall slices. The network is trained using TOC slice templates. The input to the network is eight template patterns. These are the TOC diagonal and wall slices of four segments from one transabdominal cardiac cycle. The network is trained over the eight patterns. The training terminates when the worst error in all patterns in one pass is less than 0.1. Typically the average error will be in the range of 0.001 .
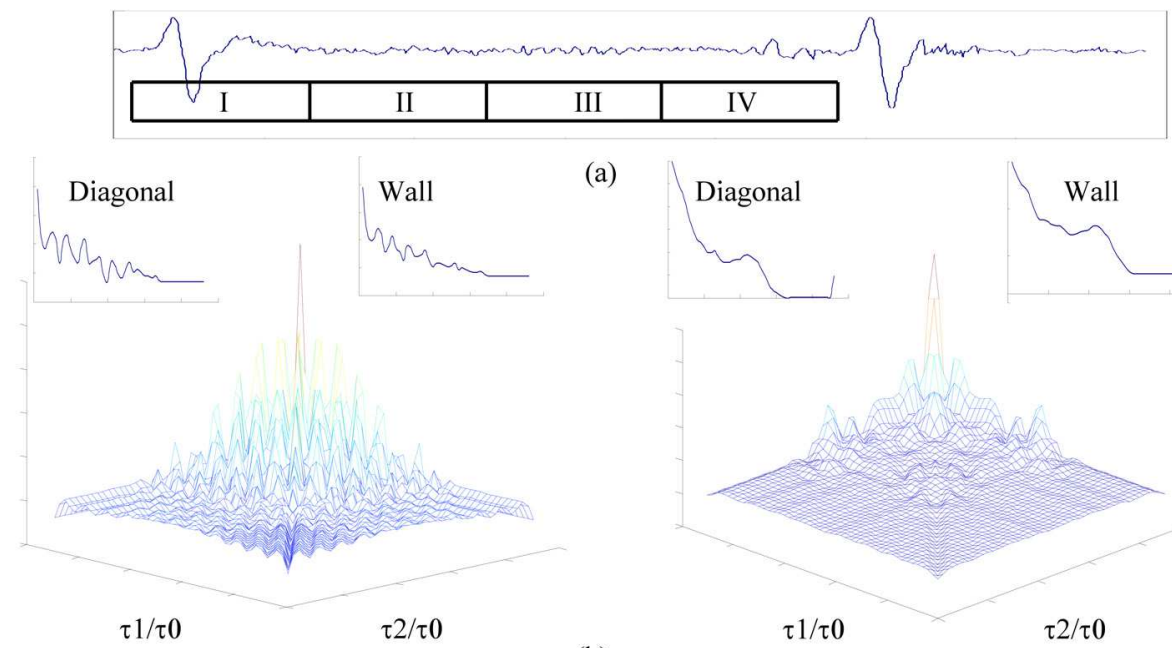

(b)

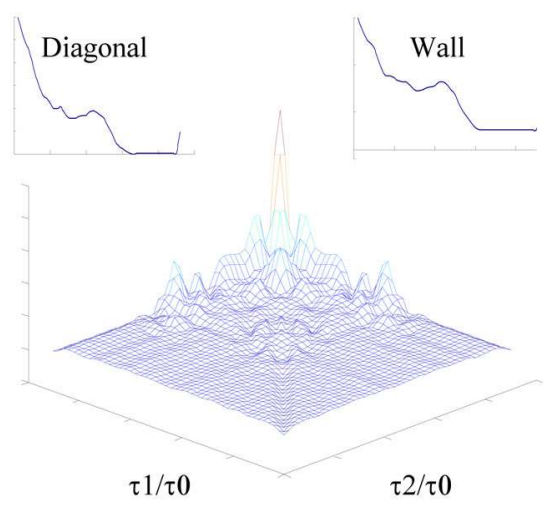

Fig. 2.1. (a) Transabdominally-measured ECG (Code: 16-23) showing segmentation (segments I, II, III, and IV, 250 msec each). (b) The TOCs and their diagonal and wall slices (insets) for the QRS-free ECG (l.h.s.) and the second fetal heartbeat with maternal contribution (r.h.s.). $\tau_{0}$, $\tau_{1}$ and $\tau_{2}$ are, respectively, the reference, first and second time lags of the TOCs. 


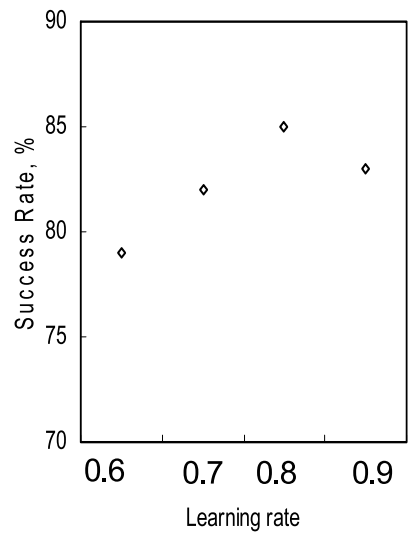

(a)

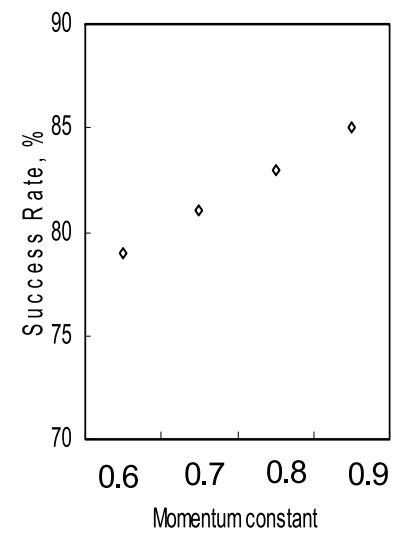

(b)

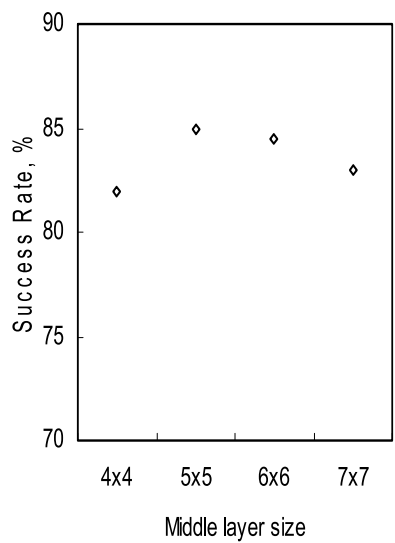

(c)

Fig. 2.2. The effect of changing (a) the learning rate, (b) the momentum constant, and (c) the middle layer size, on the classification rate of the fetal heartbeats with maternal contribution from transabdominally-measured ECG signals and employing TOC diagonal slices and their templates to be matched using a single-hidden-layer perseptron back-propagation with momentum. Segment length is $250 \mathrm{msec}$ each. The optimised parameters are: learning rate = 0.8 , momentum constant $=0.9$, and middle-layer size $=5 \times 5$.

\subsubsection{Cumulant matching of the fetal heartbeats}

Each one of the four transabdominal ECG segments (Data length $=250 \mathrm{msec}$ ) has ten corresponding templates used for matching. An optimised cubic Volterra structure is employed to synthesise the four transabdominal ECG segments and the corresponding templates.

\subsubsection{The maternal heartbeat classification rates}

The classification rate is $100 \%$ for maternal QRS-complexes using the TOC template matching technique with single-hidden-layer classification. To calculate the maternal heart rate an auxiliary method to pinpoint the R-wave employing an adaptive thresholding has been used. Note that this is not accurate when one deals with deformed QRS-complexes in heart patients. The data obtained include all mothers' ECGs exhibiting normal-to-the-patient QRS-complexes. The instantaneous maternal heart rate is calculated by dividing 60 by the Rto-R interval (in seconds). The application of this auxiliary routine leads to a maternal heart rate with an accuracy of $99.85 \%$. 


\subsubsection{Classification rate for the hybrid technique}

\section{Definitions}

1. The Sensitivity (Se) is defined as the ratio of the True Positives (TP) to the sum of the True Positives and the False Negatives (FN). The sensitivity reports the percentage of true beats that are correctly classified by the algorithm.

2. The Specificity (Sp) is defined as the ratio of the True Positives (TP) to the sum of the True Positives (TP) and the False Positives (FP). It reports the percentage of classified heartbeats which are in reality true beats.

3. The classification rate: The mean value of the sensitivity and the specificity is used as the criterion for the effectiveness of the technique.

Table 2.1 shows the fetal heart detection quality and classification rate using transabdominally-measured ECGs and their respective TOC diagonal or wall slices with and without linearisation. The combined diagonal and wall slices improve the classification rate by about $1 \%$ over and above that achieved by either slice. A further improvement of about $1 \%$ is achieved by using two off-diagonal and off-wall slices. A second-order Volterra synthesiser results in a higher detection rate of $83.49 \%$.

The highest achievable classification rate for non-invasive fetal heartbeat detection using the first hybrid system is $86.16 \%$ when a third-order Volterra synthesiser is employed in conjunction with single-hidden-layer classifiers. Note that the classification rate for coincident maternal and fetal QRS complexes is $0 \%$. The classification rate of non-coincident maternal and fetal QRS-complexes is $95.55 \%$.

\begin{tabular}{|l|c|c|c|c|c|}
\hline $\begin{array}{l}\text { TOC matching } \\
\text { template slice type }\end{array}$ & $\begin{array}{c}\text { Sensitivity } \\
\mathbf{( \% )}\end{array}$ & $\begin{array}{c}\text { Specificity } \\
\mathbf{( \% )}\end{array}$ & $\begin{array}{c}\text { Classification } \\
\text { Rate (\%) }\end{array}$ & $\begin{array}{c}\text { False } \\
\text { Positives }\end{array}$ & $\begin{array}{c}\text { False } \\
\text { Negatives }\end{array}$ \\
\hline $\begin{array}{l}\text { TOC Diagonal/Wall } \\
\text { slice }\end{array}$ & 76.24 & 79.38 & 77.81 & 24744 & 28512 \\
\hline $\begin{array}{l}\text { TOC Diagonal and Wall } \\
\text { slices }\end{array}$ & 77.13 & 80.24 & 78.74 & 23712 & 27444 \\
\hline $\begin{array}{l}\text { TOC Diagonal, wall, } \\
\text { diagonal \& wall, \& 22.5o } \\
\text { slice }\end{array}$ & 78.04 & 81.18 & 79.69 & 22584 & 26352 \\
\hline $\begin{array}{l}\text { Diagonal/Wall slice 2nd } \\
\text { order LMF Volterra }\end{array}$ & 82.37 & 84.61 & 83.49 & 18468 & 21156 \\
\hline $\begin{array}{l}\text { Diagonal/Wall slice 3rd } \\
\text { order LMF Volterra }\end{array}$ & 84.46 & 87.85 & 86.16 & 14500 & 18648 \\
\hline
\end{tabular}

Table 2.1. Fetal heart detection quality and classification rate using transabdominallymeasured ECG and their respective TOC diagonal or wall slices with and without linearisation. The total number of fetal heartbeats is 120,000 and the total number of maternal ECG recordings. is 30. The performance was assessed against synchronised fetal scalp heartbeats. All mothers were during the first stage of labour at 40 weeks of gestation. 


\section{ECG bispectrum contour classification}

\subsection{Background}

This section describes a hybrid system using the mother and fetal ECG bispectral contours (BIC), which carry the signature imprints of their respective QRS-complexes, in the signal processing phase. The classification phase employs LMS-based single-hidden-layer classifiers. The maternal chest ECGs and the fetal scalp electrode ECGs have been used as templates or the HOS representatives in the classification phase. The bispectral contour matching technique is used to identify the signatures of both the maternal and fetal QRScomplexes. It will be shown that the highest achievable Fetal Heartbeat (FHB) classification rate using the BIC template matching technique is $90.12 \%$ with reduced false positives and negatives associated with the power spectrum-based FHB classification rate of $70 \%$. Furthermore, the BIC has a marginally improved classification performance over and above the TOC during episodes of overlapping fetal QRS-complexes and maternal T-waves. This is achieved at the expense of complexity and computation time. The hybrid bispectral contour matching technique is an extension to the hybrid cumulant matching technique. Therefore, the choice of the NN classifier is based on the general discussion presented previously. Prior information remain as valuable assets and are exploited herein. It is the matching of the horizontal 2-d bispectral contours that has been used in the BIC template matching technique instead of the 1-d polar bispectral slices. Because in order to use the 1-d polar bispectrum slices effectively, one needs to use a minimum of 24 polar slices to facilitate capturing the most rapid changes in the bispectrum including null features that could be used as discriminant patterns. Whereas for BIC contours, provided that they are horizontally cut at a maximum number of 10 levels, a good quality discriminant picture can be made available for the neural network classifier. For example, it is very unlikely that maxima and troughs are missed because of any changes in their respective positions.

The same procedure of Section 2 is applied with the replacement of the third-order cumulant slices by the bispectral contours (usually 10 contours including the tip of the peak and are spaced by approximately $1 \mathrm{~dB}$ ). The CPU time for the bispectrum computation is almost twice that for cumulants and 2000 times that for individual TOC slices. The Detection key operations are exactly the same as those described in Section 2 except that the third-order cumulant slices are now going to be replaced by the bispectral contours (Zgallai, 2012).

\subsection{ECG bispectrum}

The nth-order cumulant spectrum of a process $\{x(k)\}$ is defined as the $(n-1)$-dimensional Fourier transform of the nth-order cumulant sequence. The nth-order cumulant spectrum is thus defined as (Dogan and J. M. Mendel, 1993):

$$
C_{n}^{x}\left(\omega_{1}, \omega_{2}, \ldots, \omega_{n-1}\right)=\sum_{\tau_{1}=-\infty}^{+\infty} \cdots \sum_{\tau_{n-1}=-\infty}^{+\infty} c_{n}^{x}\left(\tau_{1}, \tau_{2}, \cdots, \tau_{n-1}\right) e^{-j\left(\omega_{1} \tau_{1}+\omega_{2} \tau_{2}+\ldots+\omega_{n} \tau_{n-1}\right)}
$$

where

$$
\left|\omega_{i}\right| \leq \pi \quad \text { for } \quad i=1,2, \ldots n-1, \text { and } \quad\left|\omega_{1}+\omega_{2}+\ldots+\omega_{n-1}\right| \leq \pi
$$


The bispectrum, $\mathrm{n}=3$, is defined as:

$$
C_{3}^{x}\left(\omega_{1}, \omega_{2}\right)=\sum_{\tau_{1}=-\infty}^{+\infty} \sum_{\tau_{2}=-\infty}^{+\infty} c_{3}^{x}\left(\tau_{1}, \tau_{2}\right) e^{-j\left(\omega_{1} \tau_{1}+\omega_{2} \tau_{2}\right)}
$$

where $c_{3}^{x}\left(\tau_{1}, \tau_{2}\right)$ is the third-order cumulant sequence. The computational complexity of the bispectrum is of the order of $\mathrm{N}^{3}$.

\subsection{Typical examples of bispectra and their contours}

Linearisation is a key step in the signal processing and it is applied using an optimised third-order Volterra synthesiser to all the results included. Fig. 3.1 depict dual-band-pass filtered bispectra and their contours normalised to the maternal QRS-complex spectral peak for the transabdominally-measured ECG segments I, II, III, and IV. Segment I: predominantly maternal QRS-complex, segment II: the first fetal heartbeat with maternal contribution; segment III: QRS-free ECG, and segment IV: the second fetal heartbeat with maternal contribution. The dual-band-pass filter consists of two fifth-order Butterworth filters with cut-off frequencies of $10 \mathrm{~Hz}$ to $20 \mathrm{~Hz}$, and $25 \mathrm{~Hz}$ to $40 \mathrm{~Hz}$, respectively, a passband attenuation of $0.5 \mathrm{~dB}$, and a stop-band attenuation larger than $70 \mathrm{~dB}$. The sampling rate is $500 \mathrm{~Hz}$. Optimised Kaiser weighting coefficients are used for the fetal and maternal ECGs to enhance their spectral peaks at $30 \mathrm{~Hz}$ and $17 \mathrm{~Hz}$, respectively. The Kaiser windows are centred at frequencies of $15 \mathrm{~Hz}, 16 \mathrm{~Hz}, 17 \mathrm{~Hz}, 18 \mathrm{~Hz}$, and $19 \mathrm{~Hz}$ for the maternal QRS-complex, and at frequencies of $28 \mathrm{~Hz}, 29 \mathrm{~Hz}, 30 \mathrm{~Hz}, 31 \mathrm{~Hz}, 32 \mathrm{~Hz}, 33 \mathrm{~Hz}, 34$ $\mathrm{Hz}, 35 \mathrm{~Hz}, 36 \mathrm{~Hz}, 37 \mathrm{~Hz}$, and $38 \mathrm{~Hz}$ for the fetal heartbeat.

Fig. 3.1 (I) shows the maternal QRS-complex principal bispectral peaks and contours centred at the frequency pairs $(18 \mathrm{~Hz}, 5 \mathrm{~Hz})$ and $(18 \mathrm{~Hz}, 16 \mathrm{~Hz})$. These maternal frequency pairs with a frequency peak at $18 \mathrm{~Hz}$ slightly deviate from the actual frequency of $17 \mathrm{~Hz}$ (Rizk et al., 2000), which is due to the BIC bias. The maternal optimised Kaiser window centred at $18 \mathrm{~Hz}$ will help to detect this deviated peak. Fig. 3.1 (II) shows the first fetal heartbeat principal bispectral peaks and contours at the frequency pairs $(30 \mathrm{~Hz}, 5 \mathrm{~Hz})$, $(30 \mathrm{~Hz}, 18 \mathrm{~Hz})$, and $(30 \mathrm{~Hz}, 30 \mathrm{~Hz})$. The fetal optimised Kaiser window centred at $30 \mathrm{~Hz}$ will help to detect these peaks. Note that these peaks are sharp. Fig. 3.1 (III) shows the QRS-free ECG bispectral peak and contours centred at the frequency pair $(27 \mathrm{~Hz}, 15 \mathrm{~Hz})$. Note that the BIC of the QRS-free ECG is at approximately $-12 \mathrm{~dB}$ which is $3 \mathrm{~dB}$ and $6 \mathrm{~dB}$ lower than that of the first and second fetal heartbeats, respectively. Fig. 3.1 (IV) shows the second fetal heartbeat principal bispectral peak and contours centred at the frequency pairs $(30 \mathrm{~Hz}, 5 \mathrm{~Hz})$, and $(30 \mathrm{~Hz}, 28 \mathrm{~Hz})$. The fetal optimised Kaiser window centred at 30 $\mathrm{Hz}$ will help to detect these peaks.

\subsection{Estimation of the bispectral contour matching variance}

The variance of the BIC is defined as the expected value of the squared difference in frequency (in $\mathrm{Hz}$ ) between the computed $\mathrm{BIC}$ of the $250 \mathrm{msec}$ flag window of the transabdominal ECG signal and the computed BIC from the synchronised fetal scalp electrode ECG 250 msec window. 


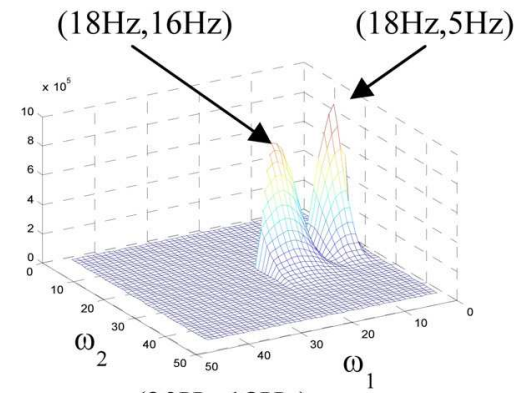

$(30 \mathrm{~Hz}, 18 \mathrm{~Hz})$
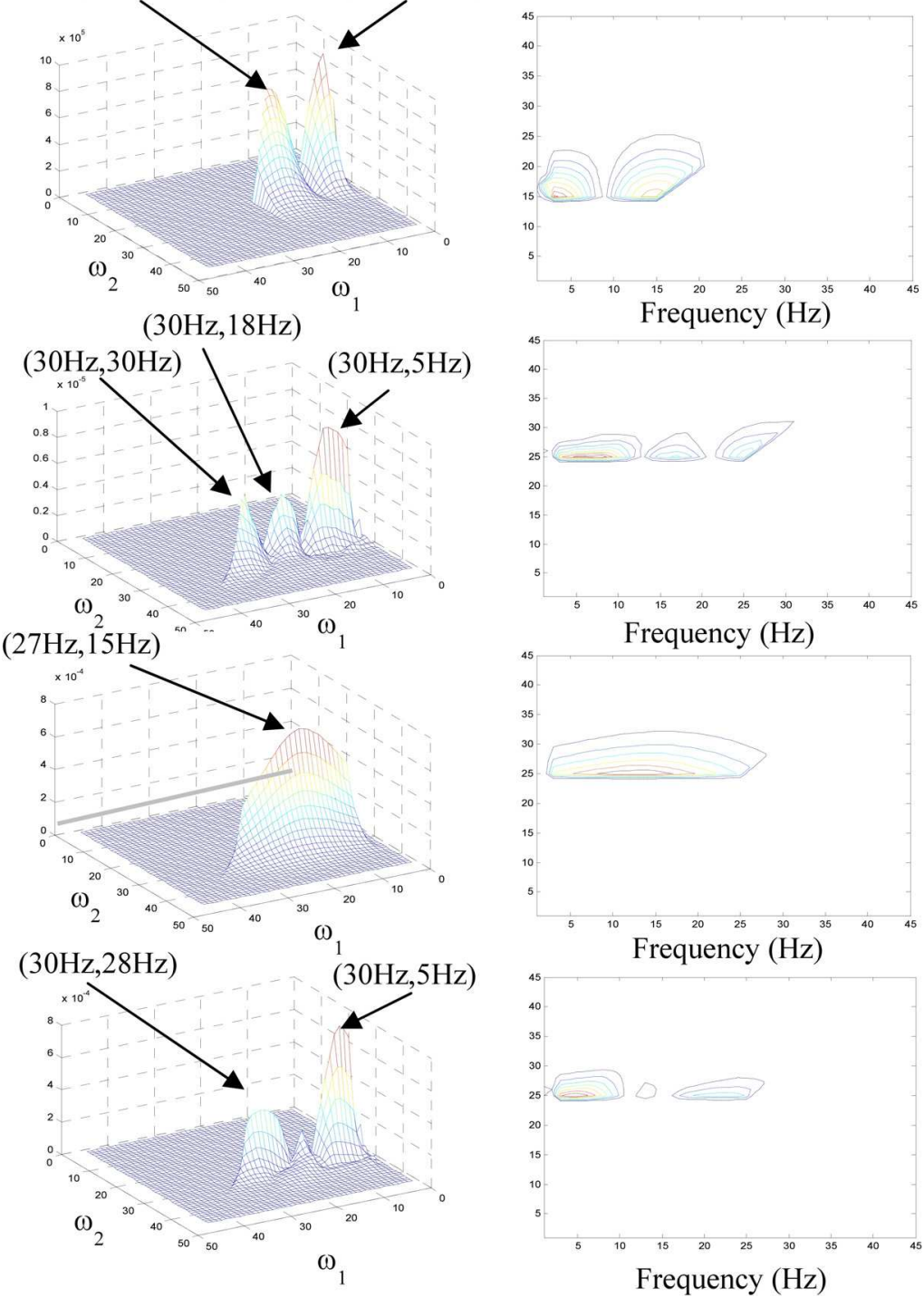

Fig. 3.1. Dual-band-pass filtered bispectra, Kaiser shaped window, (1.h.s.) and their contour maps normalised to the maternal QRS spectral peak (r.h.s.) for the transabdominallymeasured ECG segments I, II, III, and IV of Fig. 2.1. Segment I: maternal QRS-complex, Segment II: the first fetal heartbeat with maternal contribution; Segment III: QRS-free ECG; and Segment IV: the second fetal heartbeat with maternal contribution. The dual band-pass filter consists of two fifth-order Butterworth filters with cut-off frequencies of $10 \mathrm{~Hz}$ to 20 $\mathrm{Hz}$, and $25 \mathrm{~Hz}$ to $40 \mathrm{~Hz}$, respectively, and a pass-band attenuation of $0.5 \mathrm{~dB}$, a stop-band attenuation larger than $70 \mathrm{~dB}$. The sampling rate is $500 \mathrm{~Hz}$. 


$$
\operatorname{Var}_{\mathrm{b}}=\mathrm{E}\left[\left(\operatorname{Bis}\left(\omega_{1}, \omega_{2}\right) \text { Transabdominal }-\operatorname{Bis}\left(\omega_{1}, \omega_{2}\right)_{\text {fetal scalp }}\right]^{2}\right.
$$

The above variance ranges from $0.47-3.3$, average $=1.716$, when calculated for 120,000 FHBs. The variance indicates the deviation of the frequency of the BIC (in $\mathrm{Hz}$ ) of the transabdominal ECG signal from that of the fetal scalp electrode around $30 \mathrm{~Hz}$.

\subsection{The back-propagation with momentum algorithm in single-hidden-layer perceptron}

Single-hidden-layer perceptron classifiers are trained in a supervised manner with the back-propagation algorithm which is based on the error-correction learning rule. The back-propagation algorithm provides a computationally efficient method for the training of the classifiers. The back-propagation algorithm is a first-order approximation of the steepest descent technique. It depends on the gradient of the instantaneous error surface in weight space. The algorithm is therefore stochastic in nature. It has a tendency to zigzag its way about the true direction to a minimum on the error surface. Consequently, it suffers from a slow convergence property. A momentum term is employed to speed up the performance of the algorithm. The classifier used here is exactly the same as that used in Section 2.

\subsection{Optimisation of the parameters of the back-propagation algorithm}

Fig. 3.2 shows the effect of changing the learning rate $(\beta)$, the momentum constant $(\alpha)$ and the middle layer size on the classification of the maternal QRS-complexes and the fetal heartbeats using the BIC template matching technique. The effect of changing the learning rate on the classification rate is shown in Fig. 3.2 (a). Small values of $\beta$ are not able to track the variations in the bispectral contours. For classification of the bispectral contours, $\beta$ reaches its optimum value at 0.2 . For values larger than 0.2 , the output values are too large so that the difference with respect to the reference signal (template) will increase. This leads to larger error that will be fed back to the network, which will lead to slower convergence. The network will take long time to converge, or it might not converge at all. The optimum value of the momentum constant is found to be 0.2 , as depicted in Fig. 3.2 (b). Smaller values are not enough to push the adaptations to avoid local minima. While larger values tend to affect the routine detrimentally by bypassing the global minimum. The performance deteriorates significantly as the learning rate and the momentum constant diverge from their optimum values. The number and size of the middle layers were investigated by trial and error. There is a trade off between networks that should be small enough to allow faster implementation, and larger networks in size and number of hidden layers which are very slow and can not be implemented on-line using the current technology. Large networks could have complex relationships that represent nonlinearities that might not exist in the real signals at all. The optimum parameters indicated in Fig. 3.2 are calculated without considering the CPU time factor which might render those parameters undesirable for real-time applications. The CPU time for training is in the range of 17 to $60 \mathrm{sec}$. The average mean-squared error (MSE) is 0.04 . The worst error is 0.1 , which is the criterion for convergence. The implemented neural network has a single middle layer size of $6 \times 6$ as shown in Fig. 3.2 (c). The number of passes (epochs) required for training varied from 6 to 14 . 

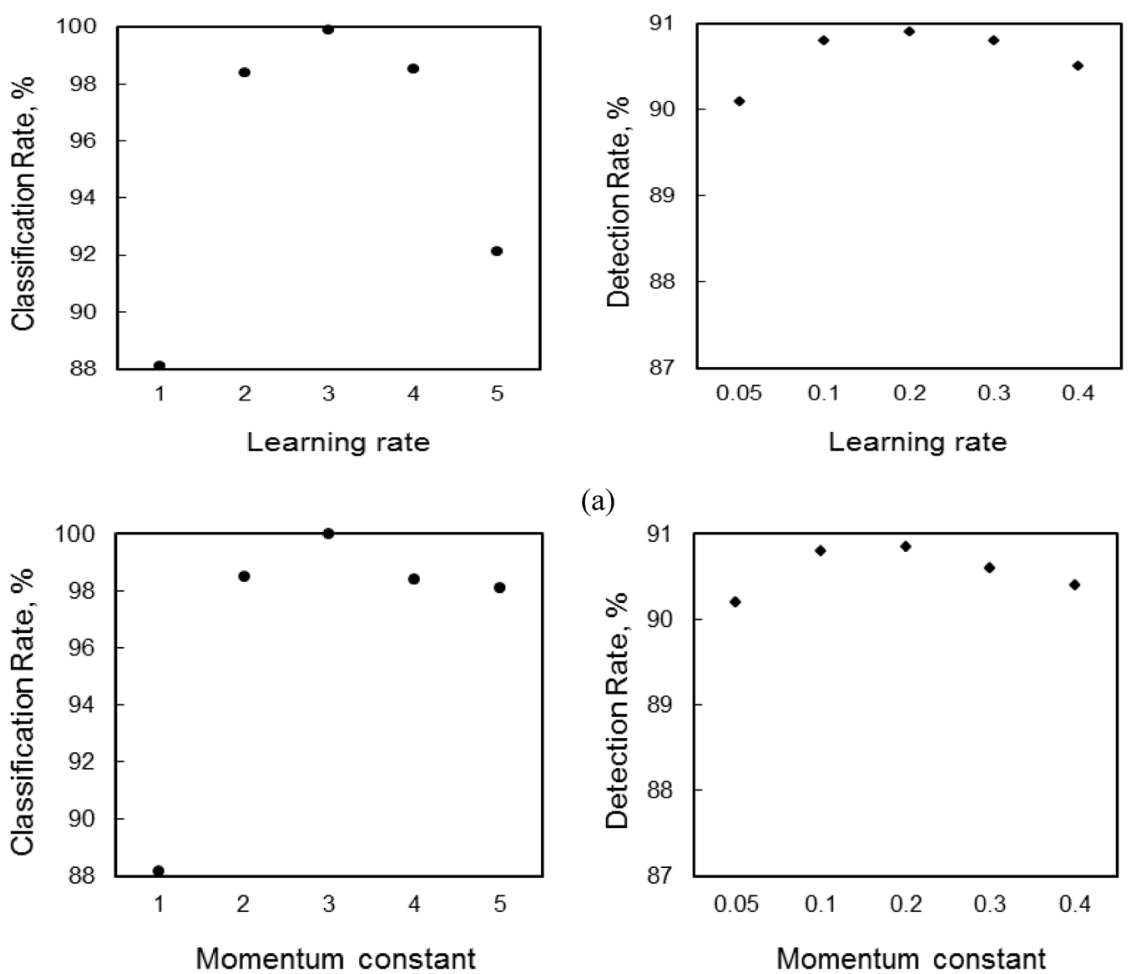

(a)

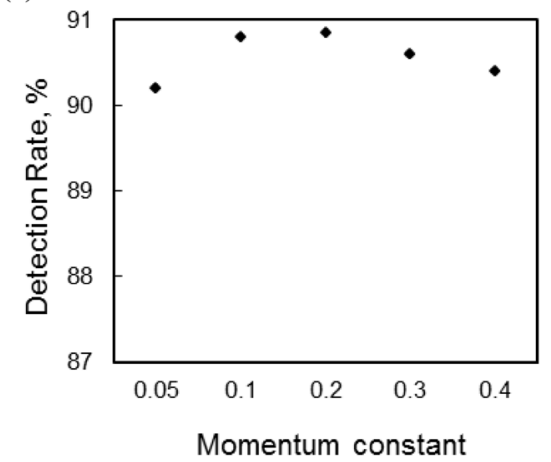

(b)

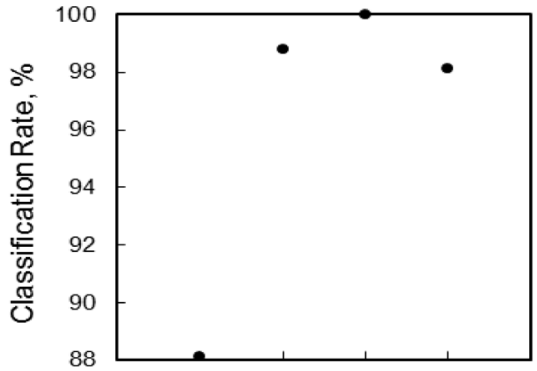

Middle layer size

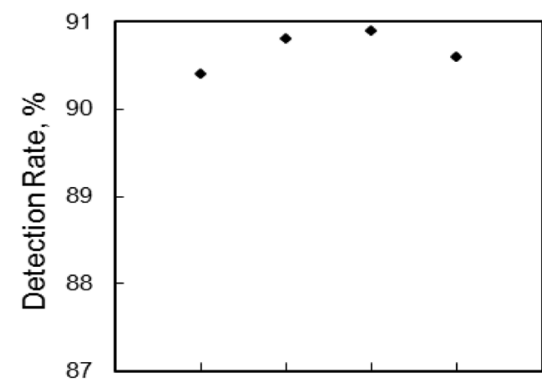

Middle layer size

(c)

Fig. 3.2. The effect of changing (a) the learning rate, (b) the momentum constant, and (c) the middle layer size on the classification rate of the maternal QRS-complexes (1.h.s.) and fetal heartbeats (r.h.s.) from transabdominally-measured ECG signals using bispectral contours and their templates to be matched using a single-hidden-layer perceptron backpropagation algorithm with momentum. Data length $=250 \mathrm{msec}$. The optimised parameters for the BIC classification are: learning rate $=0.2$, momentum constant $=0.2$, and middle-layer size $=6 \times 6$. (Code: 5-1-100). 


\subsection{Bispectral contour matching of the maternal QRS-complex and fetal heartbeat}

The bispectral contour template matching technique works with only 10 templates of maternal transabdominal QRS-complexes and 20 templates of fetal heartbeats with maternal contribution. The classification of the four segments involves a pattern-by-pattern updating rather than batch updating for the weight adjustments. This is more suitable to speed up the performance. Pattern-by-pattern updating tends to be orders of magnitude faster than batch updating. However, it should be noted that pattern-by-pattern updating is harder to parallelise (Zgallai, 2012).

\subsection{The maternal QRS-complex and the fetal heartbeat classification rates}

\subsubsection{The maternal QRS-complex classification rate}

Table 3.1 shows a top classification rate of $100 \%$ for maternal QRS-complexes using bispectral contours for signal processing and single-hidden-layer perceptron classification. The 100\% maternal QRS-complex classification rate has been achievable with or without linearisation. It makes no difference to the results. A brief description of the optimised parameters required for the linearisation process is given. To calculate the maternal heart rate an auxiliary method to pinpoint the R-wave is needed. For this application an adaptive thresholding method has been employed. Note that this is not very accurate when one deals with deformed QRS-complexes in heart patients. All maternal ECGs, however, exhibit normal-to-the-patient QRS-complexes. The instantaneous maternal heart rate is calculated by dividing 60 by the R-to-R interval (in seconds). The application of this auxiliary routine leads to a maternal heart rate with an accuracy of $99.85 \%$.

Parameters: The second-order Volterra parameters are: filter length $=6$, step-size parameters $=0.005$, and 0.0004 for linear and quadratic parts, respectively, delay $=3$. The third-order Volterra parameters are: filter length $=6$, step-size parameters $=0.001,0.0002$, and 0.0004 for linear, quadratic and cubic parts, respectively, delay $=4$. A dual-band-pass filter is applied to the bispectrum, the first has a band-pass of $10 \mathrm{~Hz}$ to $20 \mathrm{~Hz}$ and the second has a bandpass of $25 \mathrm{~Hz}$ to $40 \mathrm{~Hz}$. Optimised Kaiser windows centred at frequencies of $15 \mathrm{~Hz}, 16 \mathrm{~Hz}$, $17 \mathrm{~Hz}, 18 \mathrm{~Hz}$, and $19 \mathrm{~Hz}$ for the maternal spectrum, and at frequencies of $28 \mathrm{~Hz}, 29 \mathrm{~Hz}, 30$ $\mathrm{Hz}, 31 \mathrm{~Hz}, 32 \mathrm{~Hz}, 33 \mathrm{~Hz}, 34 \mathrm{~Hz}, 35 \mathrm{~Hz}, 36 \mathrm{~Hz}, 37 \mathrm{~Hz}$, and $38 \mathrm{~Hz}$ for the fetal spectrum are used in both the power spectrum and the BIC.

\subsubsection{Fetal heartbeat detection quality and classification rate for the bispectral contour template matching technique}

Table 3.2 summarises the results of the fetal heartbeat detection using the power spectrum method (second-order statistics), and the bispectrum contour template matching technique. Optimised adaptive LMF-based second- and third-order Volterra synthesisers are employed. The power spectrum method has a classification rate of $71.47 \%$. Using the second hybrid system, the classification rate increased to $87.72 \%$ without linearisation, and to $88.28 \%$ and $90.12 \%$ using second- and third-order Volterra synthesisers with LMF update, respectively. The second hybrid method has an improvement of $19 \%$ and $4 \%$ in the classification rate over and above that achieved with the second-order statistics and the TOC template matching technique, respectively. The classification rate of the coincident maternal and fetal QRS-complexes is $0 \%$. The classification rate of non-coincident maternal and fetal QRS-complexes is $99.21 \%$. 
Parameters:The second-order Volterra parameters are: filter length $=6$, step-size parameters $=0.005$, and 0.0004 for linear and quadratic parts, respectively, delay $=5$. The third-order Volterra parameters are: filter length $=6$, step-size parameters $=0.001,0.0002$, and 0.0004 for linear, quadratic and cubic parts, respectively, delay $=5$. A dual-band-pass filter is applied to the bispectrum, the first has a band- pass of $10 \mathrm{~Hz}$ to $20 \mathrm{~Hz}$ and the second has a bandpass of $25 \mathrm{~Hz}$ to $40 \mathrm{~Hz}$. Optimised Kaiser windows centred at frequencies of $15 \mathrm{~Hz}, 16 \mathrm{~Hz}$, $17 \mathrm{~Hz}, 18 \mathrm{~Hz}$, and $19 \mathrm{~Hz}$ for the maternal spectrum, and at frequencies of $28 \mathrm{~Hz}, 29 \mathrm{~Hz}, 30$ $\mathrm{Hz}, 31 \mathrm{~Hz}, 32 \mathrm{~Hz}, 33 \mathrm{~Hz}, 34 \mathrm{~Hz}, 35 \mathrm{~Hz}, 36 \mathrm{~Hz}, 37 \mathrm{~Hz}$, and $38 \mathrm{~Hz}$ for the fetal spectrum are used in both the power spectrum and the BIC.

\begin{tabular}{|l|c|c|}
\hline $\begin{array}{l}\text { Spectral matching template with ANN } \\
\text { classifiers }\end{array}$ & $\begin{array}{c}\text { The power } \\
\text { spectrum }\end{array}$ & $\begin{array}{c}\text { The bispectrum } \\
\text { contours }\end{array}$ \\
\hline Classification rate & 99.84 & 100.00 \\
\hline
\end{tabular}

Table 3.1. The classification rate for the maternal QRS-complex using maternal transabdominally-measured ECGs and their respective power spectrum and bispectral contours.

\begin{tabular}{|l|c|c|c|c|c|}
\hline $\begin{array}{l}\text { Spectral matching template type with } \\
\text { and without linearisation using } \\
\text { Volterra and in conjunction with } \\
\text { ANN classifiers }\end{array}$ & $\begin{array}{c}\text { Se } \\
\mathbf{( \% )}\end{array}$ & $\begin{array}{c}\text { Sp } \\
\mathbf{( \% )}\end{array}$ & $\begin{array}{c}\text { FP, } \\
\text { out of } \\
\mathbf{1 2 0 0 0 0}\end{array}$ & $\begin{array}{c}\text { FN, } \\
\text { out of } \\
\mathbf{1 2 0 0 0 0}\end{array}$ & $\begin{array}{c}\text { Classification } \\
\text { rate } \\
\mathbf{( \% )}\end{array}$ \\
\hline Power spectrum with linearisation & 71.29 & 71.44 & 34272 & 34537 & 71.37 \\
\hline $\begin{array}{l}\text { Bispectral contour without } \\
\text { linearisation }\end{array}$ & 87.97 & 87.46 & 15048 & 14436 & 87.72 \\
\hline $\begin{array}{l}\text { Linearised bispectral contour using 2nd } \\
\text { order adaptive LMF Volterra } \\
\text { synthesiser }\end{array}$ & 88.53 & 88.04 & 14352 & 13764 & 88.28 \\
\hline $\begin{array}{l}\text { Linearised bispectral contour using 3rd } \\
\text { order adaptive LMF Volterra } \\
\text { synthesiser }\end{array}$ & 90.53 & 89.73 & 12324 & 11364 & 90.12 \\
\hline
\end{tabular}

Table 3.2. Fetal heart detection quality and classification rate using transabdominallymeasured ECG and their respective power spectrum and bispectral contours with and without linearisation. The total number of fetal heartbeats is 120,000 and the total number of maternal ECG recordings is 30. The performance was assessed against synchronised fetal scalp heartbeats. All mothers were during the first stage of labour at 40 weeks of gestation.

\subsection{Discussion}

The bispectral contour matching technique is an extension to the cumulant matching technique. Therefore, the choice of the NN classifier is based on the discussion presented previously. Prior information remain as valuable assets and are very much exploited herein. It is the matching of the horizontal 2-d bispectral contours that has been used in the BIC template matching technique instead of the 1-d polar bispectral slices. Because in order to use the 1-d polar bispectrum slices effectively, one needs to use a minimum of 24 polar slices to facilitate capturing the most rapid changes in the bispectrum including null features that could be used as discriminant patterns. Whereas for BIC contours, provided that they are 
horizontally cut at a maximum number of 10 levels, a good quality discriminant picture can be made available for the neural network classifier. For example, it is very unlikely that maxima and troughs are missed because of any changes in their respective positions. Approximately 50,000 maternal cardiac cycles have been included in the analysis. The numbers of bispectral contours compound templates are 10 for the maternal chest, 10 for the fetal scalp, and 140 for the transabdominally-measured $250 \mathrm{msec}$ segments, respectively. Each bispectral compound template is made of 10 horizontal templates at different levels. Starting from a normalised $0 \mathrm{~dB}$ and going down in steps of $1 \mathrm{~dB}$ each to a $-10 \mathrm{~dB}$.

The maternal transabdominal ECG signal is linearised using an optimised LMF-based secondor third-order Volterra synthesiser. The second-order Volterra synthesiser parameters are: filter length $=6$, step-size parameters $=0.005$, and 0.0004 for linear and quadratic parts, respectively, delay $=5$. The third-order Volterra synthesiser parameters are: filter length $=6$, step-size parameters $=0.001,0.0002$, and 0.0004 for linear, quadratic and cubic parts, respectively, delay $=$ 5. The transabdominal ECG signal is segmented into four segments containing; (I) The maternal QRS-complex, (II) the first fetal heartbeat with maternal contribution, (III) QRS-free ECG, and (IV) the second fetal heartbeat with maternal contribution. To segment the transabdominal ECG signals, the window length is carefully chosen to; (i) Yield an acceptable upper threshold of both the deterministic and stochastic noise types inherent in the higherorder statistics of the ECG signals encountered, and (ii) allow the detection of one, two, three, or four fetal heartbeats (FHBs) within one maternal transabdominal cardiac cycle.

The classification procedure starts by matching the bispectral contours of the segments to those of the templates until the first and the second maternal QRS-complexes are detected and their R-waves are pinpointed. The maternal heart rate is accurately calculated from the knowledge of the current and previous R-wave positions. Then, the search for the fetal heartbeat starts at $50 \mathrm{msec}$ before the first maternal R-wave and continues until the second maternal R-wave is reached. Although the ECG bispectral contour template matching technique is very effective in detecting the occurrence of the fetal heartbeats as a whole in the frequency domain even when it is completely buried in noise, it cannot locate the R-wave in the time domain over a window length of $250 \mathrm{msec}$. However, the maternal heartbeats can be measured fairly accurately and calculate the instantaneous heart rate for the mother. Hence, by counting the number of fetal heartbeats that have occurred between two successive maternal R-waves, one can easily calculate the averaged FHR within the maternal cardiac cycle;

The average FHR = MHR $\times$ Number of FHBs / number of maternal heartbeats

the instantaneous maternal heart rate is previously known with some degree of accuracy, and the relative fetal to maternal heartbeat is also known within the maternal cardiac cycle. Hence, the averaged fetal heart rate can be calculated within each maternal cardiac cycle.

\subsubsection{The effect of window length on the bispectral contour variance}

The variance of the bispectrum for the optimum window length of 250 msec with FHB occurrence ranges from 0.47 to 3.3 with an average value of 1.716 . The variance of the bispectrum is smaller than that of the third-order cumulants. A further $15 \%$ increase in the variance of the bispectrum is due to an increase in the maternal heartbeat from $60 \mathrm{bpm}$ to $100 \mathrm{bpm}$. The latter has resulted in an 30\% decrease in segment size. 


\subsubsection{Parameters of the single-hidden layer perceptron}

The network has been optimised in terms of its learning rate, momentum constant, and hidden layer size to achieve the minimum mean-squared error. The optimum learning rate is found to be 0.2 . The optimum momentum constant is found to be 0.2 . The single-hidden-layer has an optimum dimension of $6 \times 6$. The input to the first layer is the bispectral contours of the four transabdominally-measured ECG segments. The network is trained using the BIC templates. During the training phase, the input to the network is four template patterns. These are the BIC of four segments from one transabdominal cardiac cycle. For example the first is the maternal QRS-complex BIC, the second is the first fetal heartbeat BIC, the third is the QRS-free ECG BIC, and the fourth is the second fetal heartbeat BIC. The network is trained over ten templates of each of the four segments. The training terminates when the worst error in all patterns in one pass is less than 0.1. Typically the average error will be in the range of 0.001 .

\section{ECG abnormality classification using polyphase}

The main motivations behind the use of HOS are their ability to (i) preserve the phase of the signal frequency components (ii) suppress Gaussian noise, (iii) characterise and separate motion artefact (Nikias and Petropulu, 1993), and other non-Gaussian low frequency components, and (iv) detect and classify non-linearities. HOS algorithms are employed to develop discriminant contour patterns in the multi-dimensional phase of the polyspectra (polyphase) of normal looking ECGs in outpatients having weariness and general malaise or have recently had suspected angina. Similar polyphase patterns have been found in the ECGs of acute myocardial infarct patients with or without diagnostic S-T segment and T-wave changes. The polyphase computation is done in milliseconds but a temporal window of 10 ECG cycles is necessary in each of the polyspectral averaging process. The polyphase patterns can be displayed every 10 seconds. A high resolution three-lead ECG is adequate for polyphase discrimination. The results of a pilot study show that this is a potential diagnostic technique. Particularly in those 50\% Pre-Infarction Syndrome cases which show perfectly normal 12-lead ECGs and will not be identified early enough to prevent them from developing acute myocardial infarction (AMI), (Struebe and Strube, 1989). The standard 12-lead ECG has poor sensitivity for the early detection for (AMI). Only 40-50\% patients presenting with AMI show S-T segment and T-wave changes on the initial ECG. The rest might not receive the benefit of acute interventional therapies in the first hours after the event. The application of HOS to ECGs has shown fruition in positively identifying ischemic heart diseases (Strube and Strube, 1989, Zgallai, 2011a, Zgallai, 2011b, and Zgallai 2011c). The methodology involved decomposing the ECGs into linear and several non-linear component waves prior to identification and classification. Methods of validation employed clinical diagnoses and other apriori clinical information spanned over a number of years. Using other HOS discriminant patterns, the focus is on the identification of coronary artery disease, solely from its polyphase contour patterns. Polyphase patterns are obtained using the standard three-lead ECG, a multi-channel low-noise amplifier, an interface card and a PC. Sampling frequency is $0.5 \mathrm{KHz}$ and a resolution of 12 bits. Recurrent neural networks have been used to classify the patterns in Fig $4.1 \mathrm{c}$ and d, respectively (Rizk et al., 1999b). The employed neural network has an input layer, a middle layer, and an output layer. The input layer has 64 neurons, the middle layer has 16 neurons, and the output layer has 8 neurons. The network size has been optimised using trial and error with an MSE threshold of 0.001 . The learning rate and momentum constant are, respectively, 0.7 and 0.9 . 


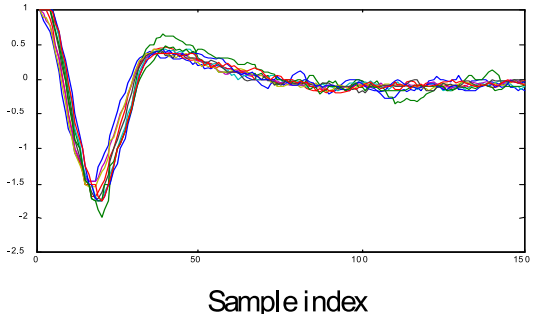

(a)

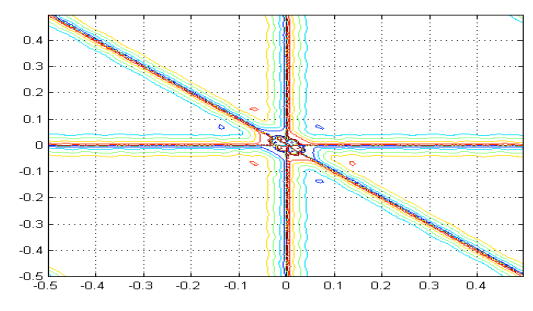

Frequency $(\mathrm{Hz})$

(c)

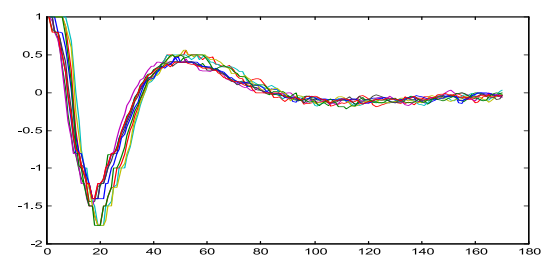

Sample index

(b)

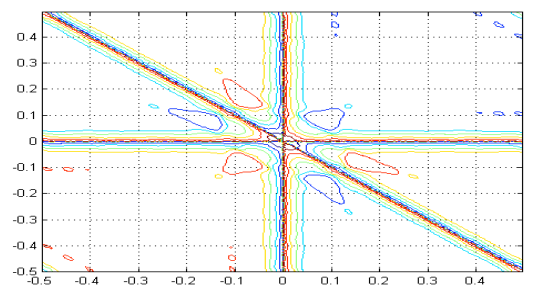

Frequency $(\mathrm{Hz})$

(d)

Fig. 4.1. Typical electrocardiogram biphase signature of an Acute myocardial infarction (AMI) presented with a normal looking 12-lead ECG without S-T segment and T-wave changes and normal sinus rhythm. (a) and (b) are ECGs of normal and AMI subjects, respectively. (c) and (d) are the corresponding bispectral phases using the direct method with frequency domain smoothing and Kaiser window. The bispectrum is the average of 10 ST bispectra, the S-T segment is 150 samples starting from the R peak (Rizk et al., 1999b).

\section{ECG abnormality classification using cumulants}

This section describes a simple and accurate multi-layer recurrent neural network classifier specifically designed to successfully distinguish between normal and abnormal higher-order statistics features of electrocardiogram (ECG) signals. The concerned abnormality in ECG is associated with ventricular late potentials (LPs) indicative of life threatening heart diseases. LPs are defined as signals from areas of delayed conduction which outlast the normal QRS period (80-100 msec). The QRS along with the P and T waves constitute the heartbeat cycle. This classifier incorporates both pre-processing and adaptive weight adjustments across the input layer during the training phase of the network to enhance extraction of features pertinent to LPs found in 1-d cumulants. The latter is deemed necessary to offset the low SNR ratio in the cumulant domains concomitant to performing short data segmentation in order to capture the LPs transient appearance. The procedures of feature selection for neural network training, modification to the back propagation algorithm to speed its rate of conversion, and the pilot trial results of the neural ECG classifier are summarised.

\subsection{Background}

The relationship between myocardial infarction (MI) and short-duration high-frequency components occurring around the terminal end of the QRS complex in the cardiac cycle of 
the ECG has been investigated by a number of dedicated research workers (Gomis et al., 1997). The high frequency components are associated with late potentials (LPs) emanating from areas of delayed conduction and outlast the normal QRS period (80-100 msec). LPs are linked with malignant ventricular tachycardia (VT) after a myocardial infarction (dead zone or scar tissue in the ventricular muscle). The later is highly correlated with sudden cardiac death. Common methodology for detecting LPs in the time domain involves temporal scanning of the S-T region of the cardiac cycle and relies upon accurate identification of the QRS boundaries ( $\mathrm{Li}$ et al., 1995). The detection problem is exacerbated by the fact that LP's are relatively weak (mv) and often below the noise floor. In the frequency domain, secondorder statistics can offer a limited success. The shapes of power spectra of normal and abnormal (malignant VT) ECGs are invariably broadly similar and without significant features above the noise floor, at approximately -70 dB (Rizk et al., 1998). LPs are essentially non-linear transient events (Gomis et al., 1997) and consequently interact with the inherent non-linearity of the cardiac waves as well as certain class of recursive non-linearly attributed to external factors such as motion artefact (Zgallai et al., 1997).

Previous work (Zgallai et al., 1997) showed that results obtained using HOS offer some empirical evidence that: (i) ECG signals contain intrinsic as well as quadratic and higherorder non- linearities, (ii) the QRS wave is predominantly linear non-Gaussian, the P and T waves are characterized by having quadratic and cubic non-linearities, (iii) the QRS wave can be totally resolved from the motion artifact in the bispectrum domains and (iv) disproportionately high-frequency non-linearity in the bicoherence squared is indicative of abnormality in an otherwise innocent looking ECG. However, non-linear filtering and a high resolution technique such as the spectral MUSIC incorporates an optimised window must be applied to a short duration data sample (without compromising the variance), prior to the application of HOS (Zgallai et al., 1997). Third-order Volterra filtering applied to raw data can be beneficial in isolating quadratic and cubic non-linearity in the higher-domains (Zgallai et al., 2007).

The higher-order statistical features are selected and enhanced using sampled weights of a non-linear function based on a priori information about distinguished abnormality signatures in the higher domains. The function is modified adaptively during the training of the neural network which employs ten 1-d cumulants every 1000 or less cycles per patient. After this the updated version of the function parameters are fixed over the next 1000 cardiac cycles. Subsequently, a simple neural network classifier based on a modified version (Jacobs, 1988, and Hush and Salas, 1988) of the back-propagation algorithms performs accurate LPs and even ischemic ECG classification (Zgallai, 2011 a, Zgallai, 2011 b, and Zgallai, 2011 c).

\subsection{Higher-order statistics feature selection and enhancement}

The experimental setup consists of an ECG monitor, interface card and a workstation. Raw ECG data are measured using three orthogonal surface electrodes, sampled at $500 \mathrm{~Hz}$ and fed to the computer which performs the following operations. Accurate on-line QRS detection. This involves Volterra whitening filters in the time domain or / and the highresolution spectral MUSIC in the frequency domain. Positions of ECG peaks are pinpointed in the time domain. The MUSIC algorithm incorporates two sliding sets of three overlapping Kaiser windows and adaptive thresholding operations which not only 
pinpoint the high-level low-frequency QRS spectral peaks (LFQRSSPs) per cycle, but also performs the preliminary spotting of the low-level high-frequency late potential components over a range of frequencies from 100-250 Hz. Detection of late potential highfrequency spectral peaks is carried out off-line every 5 LFQRSSPs to allow appropriate segmentation between the R-R marking in the time domain processing which runs almost synchronisingly with the MUSIC routine. A detailed procedure for segmentation involves calculating the bicoherence squared and mapping a particular region for each individual segment to confirm existence of quadratic non-linearity before moving on to interrogate another segment or skip a few segments up to the next $\mathrm{R}$ peak. This controlled skipping helps to avoid the highly non-linear $\mathrm{T}$ wave of the present cycle and the $\mathrm{P}$ wave of the adjacent one.

The Volterra filtering can be used to partially suppresses motion artifact only in those cases of missing LFQRSSPs and the MUSIC routine is repeated over the same cardiac cycle for confirmation of the presence or absence of QRSs. This has been found to be necessary in extreme cases and in the absence of QRS waves (ventricular fibrillation). Offline calculations of the cumulant diagonal and wall 1-d slices are performed on those segments suspected of having LPs as depicted in Fig. 5.1. It is clearly seen that abnormality is manifested in the eminent petal pattern (a horizontal slice has a petal shape) in the cumulant domains. Five thousand cardiac cycles of normal and abnormal ECGs were put to the test. An arbitrarily chosen non-linear function modifies the envelope of the so-called 'petal pattern' to enhance its peculiarity against background artifact. The non-linear function is then sampled across the input layer of the neural network.

\subsection{Design of the neural classifier}

Fig. 5.2 shows the network preceded by a preprocessing unit which performs the task of determining a set of meaningful and representative features in the HOS domains. A sigmoid logistic function is used to describe the input-output relation of the non-linear device. The neural network is designed to classify two classes; normal and abnormal third-order cumulants. The combined use of skewness and kurtosis can provide more accuracy in difficult cases.The utility of the diagonal slice of the fourth-order cumulant can be of more help when used in the desired response for the third output. The use of higher than the third-order statistics adds more complexity to the network.

\subsubsection{Block adaptive weight adjustment}

Initially the classification was attempted by feeding cumulant slices of short ST segments of the order of 10 to 30 samples at $500 \mathrm{~Hz}$ sampling rate. This attempt was $80 \%$ successful as the network missed low profile petal patterns with low levels of signal-to-noise ratios in their vicinity as a result of short data segmentation. A function was introduced to strengthen the relative magnitude of the discriminant cumulant slice features. The function is sampled across the input layer and its parameters $(\alpha, \beta)$ can be adaptively changed for each cumulant slice fed during the training phase which usually takes up to 10 modified cumulant slices every 1000 cardiac cycles. Obviously the shape of function can be changed to cater for other types of abnormalities. 


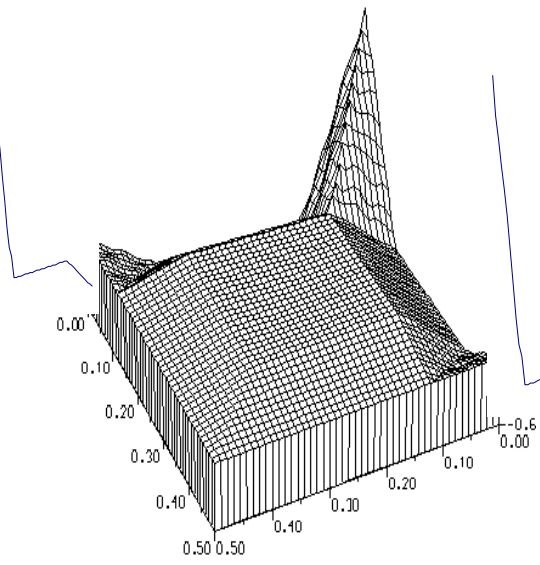

(a)

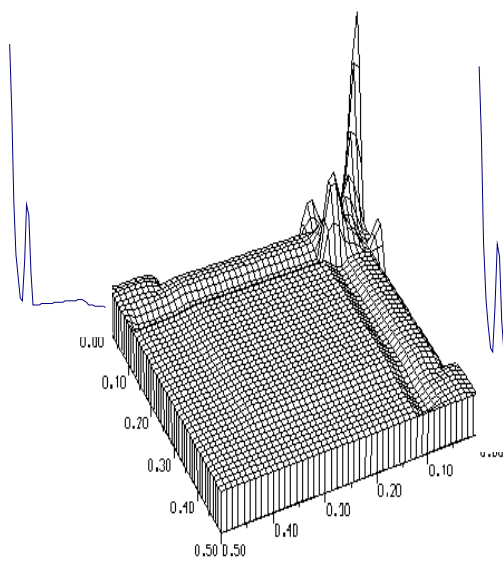

(b)

Fig. 5.1. Typical third-order cumulants and their 1-d diagonal and wall slices shown in insets (left, right) of (a) a normal subject and (b) a subject having infarction in the ventricular muscle.

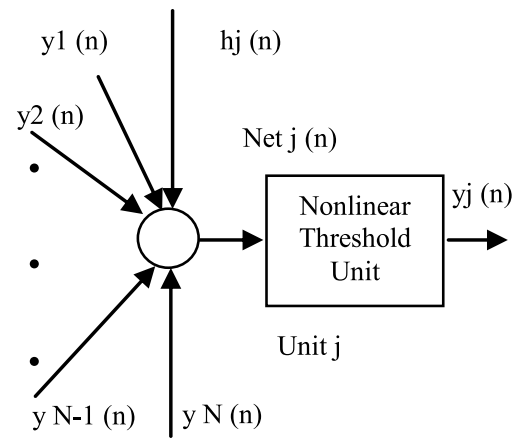

(a)
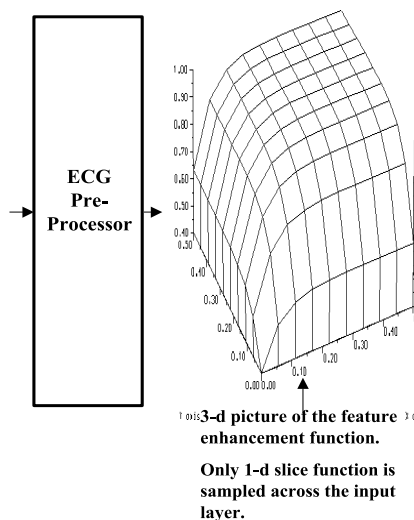

layer.

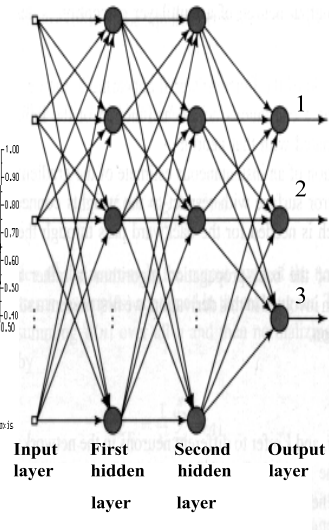

(b)

Fig. 5.2. Architecture of the four-layerd neural network. (a) Neuron or processing unit in the network. (b) The four-layer neural network. Only 1-d slice of the weight function modifies the corresponding cumulant slice.

\subsubsection{Modification to the back-propagation algorithm}

The back propagation method (Hush and Salas, 1988) used in the supervised learning of a multi-layer neural network is basically a gradient descent method. Although this method 
has become the most popular learning algorithm for multi-layer networks, its rate of convergence is often found to be too slow for practical applications. Well established methods have been adopted (Hush and Salas, 1988). In the modified back-propagation method, every weight $\mathrm{w}_{\mathrm{ij}}$ in the network is given its own learning rate $\eta_{\mathrm{ij}}$, and the training data set is divided into a number of epochs each containing $\mathrm{K}$ training patterns (training patterns are 1-d cumulants from overlapping segments of the ST region). The weight $\mathrm{w}_{\mathrm{ij}}$ and learning rate $\eta_{\mathrm{ij}}$ are updated every time after an entire training epoch (10 cumulants) has been presented to the network. The weight and learning rate updating rules of the modified back-propagation algorithm can be summarized as follows (Hush and Salas, 1988).

$$
\begin{gathered}
w_{i j}(n+1)=w_{i j}(n)+\eta_{i j}(n+1) \sum_{k=1}^{K} \delta_{k j}(n) y_{k i}(n)+\tau \Delta w_{i j}(n-1) \\
\eta_{i j}(n+1)=\eta_{i j}(n)+\Delta \eta_{i j}(n) \\
\Delta \eta_{i j}(n)=\left\{\begin{array}{lll}
\Omega, & \text { if } & S(n-1) D(n)>0 \\
-\phi \eta_{i j}(n), & \text { if } & S(n-1) D(n)<0 \\
0, & \text { otherwise }
\end{array}\right\} \\
S(n)=(1-\Theta) D(n)+\Theta S(n-1) .
\end{gathered}
$$

The index $\mathrm{n}$ refers to the $\mathrm{nth}$ epoch in the training data; the index $\mathrm{k}$ refers to the $\mathrm{kth}$ pattern in an epoch containing K patterns; $\delta_{\mathrm{kj}}$ is the modulated error signal of neuron $\mathrm{j}$ with the kth pattern in an epoch; $y_{k i}$ refers to the actual computed output of neuron I with the kth pattern in an epoch; $\varepsilon_{\mathrm{k}}$ is the index performance to be minimised by the weight update rule with the kth input pattern; finally, $\Omega, \Phi$, and $\Theta$ (all of which have values between 0 and 1 ) are the control parameters.

\subsection{Experimental results}

Three orthogonal leads ECG were recorded from several subjects confirmed of having VT with a prior myocardial infarction (MI). Two subjects suspected of having MI but time- and frequency-domains analysis had not shown any abnormality, and several normal subjects. A total of 3,000 cardiac cycles for this pilot study. Their feature extraction and enhancement were performed. The parameter $\alpha$ and $\beta$ of the exponential weight function applied across the input layer were chosen to fall in the region of $1-2$ and $0.25-0.5$ for $\alpha$ and $\beta$, respectively. The initial learning rates $\eta_{\mathrm{ij}}(0)$ were all chosen to be 0.06 . The momentum factor, $\alpha$, was fixed at 0.09 . The control parameters $\beta, \Phi$ and $\Theta$ were chosen to be $0.03,0.1$ and 0.5 respectively. The classifier described here achieved very high $(90 \%)$ classification rate. The remaining $10 \%$ failure mainly arose because the MI suspected cases were not invasively examined and confirmed. 


\section{Statistical analysis of ECG detection methods}

ECG signals are predominantly non-Gaussian (Rizk and Zgallai, 1999 and Rizk et al., 1995), and exhibit quadratic and higher-order non-linearities supported by third- and fourth-order statistics, respectively. ECG signals do contain measurable quantities of quadratic and cubic non-linearities. Such quantities if not synthesised and removed before any further processing for the purpose of signal identification and classification could lead to poor performance with regard to fetal QRS-complex detection rates. The non-linearity in the ECG signal can be detected using the bicoherence squared. The bicoherence squared has peaks at the frequency pairs of $(6 \mathrm{~Hz}, 15 \mathrm{~Hz})$ and $(14 \mathrm{~Hz}, 14 \mathrm{~Hz})$ for the fetal scalp cardiac cycle, $(15$ $\mathrm{Hz}, 15 \mathrm{~Hz})$ for the maternal chest cardiac cycle, and $(7.5 \mathrm{~Hz}, 7.5 \mathrm{~Hz})$ for the maternal transabdominal cardiac cycle. These bicoherence peaks support non-linearity (Zgallai, 2007). There is a general consensus that individual cardiac cycles are locally stationary. However, when applying a highly dimensional signal such as the transabdominal ECG that have several individual non-linear and deterministic signals overlapping both in the time and frequency domains, all coexisting in a cocktail of noise and motion artefact, it is prudent to re-examine the validity of the stationarity assumption in relation to such signals. It is only natural to expect that the proximity of two non-linear signals such as the maternal and fetal QRS-complexes would result in non-linear (quadratic and higher-order) coupling and this in turn would invoke non-stationarity. The bispectral OT region is insepcted (Nikias and Petropulu, 1993) for the maternal bispectral contour maps at a level of $-30 \mathrm{~dB}$. When the two R-waves of the maternal and fetal QRS-complexes are separated by $200 \mathrm{msec}$, the resultant bispectrum does not support the OT region (Zgallai, 2007). However, the situation is totally different when the two R-waves are as close as $35 \mathrm{msec}$. The OT region of the bispectrum is fully occupied and non-stationary (Zgallai, 2007). Hence, conventional signal processing techniques to separate the maternal and fetal QRS-complexes cannot be used. This problem has been adequately solved by linearising (at least removing quadratic coupling) the transabdominal signal before attempting to separate individual QRS-complexes.

Correlartion-based second-order statistics do not show any distinguishable features that could be used to differentiate between maternal QRS-complex, fetal heartbeat with maternal contribution, and QRS-free ECG contributions. The FFT method reveals a fetal scalp electrode ECG principal spectral peak at $30 \mathrm{~Hz}$ (Zgallai, 2007). The FFT method for the transabdominal cardiac cycle reveals the maternal principal spectral peak of $15 \mathrm{~Hz}$ (Zgallai, 2007). However, the FFT does not clearly show fetal spectral peak from the segmented transabdominal signal. There could be a shallow peak as low as $28 \mathrm{~Hz}$ or a shifted peak as high as $42 \mathrm{~Hz}$ (Zgallai, 2007).

Statistical analysis of ECG data, including Pearson's correlation analysis and higher order moments have been carried out (Rizk and Zgallai, 1999). The value of Pearson's productmoment correlation coefficient for both the third-order cumulant and the bispectral contour method is within the range of -0.1 to +0.1 .

The Receiver Operating Characeteristics (ROC) analysis has been used to statistically analyse the results of the two propsoed detection methods, third-order cunulant and bispectral contour, compared to the second-order statistics method. The Area Under Curve (AUC) has been used as a measure for diagnostic accuracy and discriminating power. The second-order statistics-based, third-order cumulant slice, and the bispectral controue 
methods have AUC values of $0.731,0.794$, and 0.843 , respectively. This suggests that the third-order cumulant is a better detection method than second-order statistics-based, and that the bispectral contour method outperforms the third-order cumulant method.

Youden's index, defined as sensitivty + specificity -1 , has also been used for the detection methods. The second-order statistics-based methods have indices in the range of 0.42 to 0.55 . The third-order cumulant method has an index of 0.72 . The bispectral contour method has an index of 0.80 . This suggests that the third-order cumulant is a better detection method than second-order statistics-based, and that the bispectral contour method outperforms the third-order cumulant method.

Also, the Partial Area Under Curve (PAUC) measure has been used for a False-Positive Rate (FPR) of $10 \%$ and sensitivty larger than $75 \%$. The second-order statistics-based method gives a PAUC of 0.043 . The third-order cumulant method has a corresponding value of 0.125 whilst that of the bispectral contour method is 0.137 . This suggests that the third-order cumulant is a better detection method than second-order statistics-based, and that the bispectral contour method outperforms the third-order cumulant method.

\section{Conclusion}

The sensitivity, specificity and classification rate for the third-order slice cumulant matching hybrid system have been calculated. The technique has been evaluated for diagonal, wall, or arbitrary TOC slices, employing both the LMF-based quadratic and cubic Volterra filters. The results indicate that a linear combination of diagonal and wall slices of the TOC can improve the detection rate by up to $1 \%$ over and above the $77.8 \%$ obtainable using only either slice. Using two more arbitrary slices off-diagonal and off-wall would result in a further improvement of up to $1 \%$. Using two slices instead of only one results in an two-fold increase in the CPU time of $1 \mathrm{msec}$ using Unix WS. Further improvement of $6 \%$ to $8 \%$ is attainable with maternal transabdominal ECG signal linearisation employing second- and third-order Volterra synthesisers, respectively. Based on the first hybrid system using TOC slices for signal processing and subsequent single-hidden-layer classification, $100 \%$ and 86.16\% classification rates have been achieved for maternal QRS-complex and fetal heartbeats, respectively. Note that the classification rates for coincident and non-coincident maternal and fetal QRS-complexes are $0 \%$ and $95.55 \%$, respectively. The remaining undetected $13.84 \%$ fetal heartbeats include $9.8 \%$ overlap with the maternal QRS-complexes and $4 \%$ occur during depolarisation of the maternal T-waves. Those events unavoidably lead to significant distortion of the fetal TOCs. This means that the cumulant signatures will not be close to the TOC template signature stored in the database. Examples of false negatives and false positives have been found in the following cases, respectively, (i) a fetal heartbeat with maternal contribution TOC diagonal slice was wrongly matched to a QRSfree ECG TOC diagonal slice template, and (ii) a QRS-free ECG TOC diagonal slice was wrongly matched to a fetal heartbeat with maternal contribution TOC diagonal slice template.

Results obtained for the bispectral contour matching hybrid system from 30 cases using the non-invasive transabdominally-measured ECG signal, with the simultaneous fetal scalp electrode ECG signal as a reference, show that the method has a classification rate of $100 \%$ for normal, healthy maternal QRS-complexes and $90.12 \%$ for fetal heartbeats. It has been 
shown that an improvement of $1 \%$ to $3 \%$ is attainable with ECG signal linearisation employing second- and third-order Volterra synthesisers, respectively. Conventional methods (based on the power spectrum) of fetal heartbeat detection have a success rate in the range of $70 \%$. The second hybrid system has a significantly higher classification rate. The classification rate of fetal heartbeats for non-coincident maternal and fetal QRScomplexes is $99.21 \%$. The classification rate of fetal heartbeats for coincident maternal and fetal QRS-complexes is 0\%. This means that the hybrid bispectral contours technique fails to resolve the fetal beat when both the mother and fetal QRS-complexes are synchronised. The bispectral contour template matching technique improved the classification rate by approximately $4 \%$ over and above that of the third-order cumulant template matching technique. The difference in performance is not due to better resolvability of the latter over the former in the case of coincident maternal and fetal QRS-complexes, as both techniques fail in this respect. But, it is due to the fact that the BIC template matching technique can resolve a few of the fetal QRS-complexes occurring within the T-wave region of the mother.

Non-invasive classification of a particular type of ECG abnormality, late potentials, was investigated. This has been achieved by the prudent use of their third-order cumulant 1-d slices. A four-layer neural network classifier based on modified back-propagation algorithm and incorporating adaptive feature enhancement weights applied to its input layer during its learning phase has been successfully tested. Classification rate obtained from 3000 cardiac cycles of normal, confirmed, and suspected abnormal subjects is $90 \%$. In a separate study conducted on the same data a sophisticated recurrent back-propagation network achieved less that $80 \%$ success rate. However, the instability issues of the latter network have not been fully investigated.

\section{References}

Bentley, J. M., Grant, P. M., and McDonnel, J. T. E. (1998). Time-frequency and time-scale techniques for the classification of native and bioprosthetic heart valve sounds. IEEE Transactions on Biomedical Engineering, Vol. 45, pp. 125-128.

Bergveld, P. and Meijier, W. J. H. (1981) A New Technique for the Suppression of MECG. IEEE Transactions on Biomedical Engineering, vol. 28, pp. 348-354.

Brockett, P. L., Hinich, M. J., and Patterson, D. (1988) Bispectral-Based Tests for the Detection of Gaussianity and Linearity in Time Series. Journal of the American Statistical Association, vol. 83, No. 403, pp. 657-664.

Caudill, M. \& Butler, C. (1992) Understanding Neural Networks: Computer Explorations, MIT press.

Crowe, J. A. et al., (1995) Sequential recording of the abdominal fetal electrocardiogram and Magnetogram. Physiological Measurements, vol. 16, pp. 43-47.

De Lathauwer, K., De Moor, B., and Vandewalle, J. (2000) Fetal electrocardiogram extraction by blind source separation. IEEE Transactions on Biomedical Engineering, Vol. 47, No. 5, pp. 567- 572, May.

Dogan, M. C. and Mendel, J. M. (1993) Antenna Array Noise Re-conditioning by Cumulants. Proceedings of the IEEE Signal Processing Workshop on Higher Order Statistics, CA.

Gomis, P. et al., (1997). Analysis of abnormal signals within the QRS complex of the highresolution electrocardiogram. IEEE Transactions on Biomedical Engineering, Vol. 44, No. 8, pp. 681-693, August.

Herbert, J. M. et al., (1993) Antepartum Fetal Electrocardiogram Extraction and Analysis. Proceedings of Computers in Cardiology, pp. 875-877. 
Hush, D. R., and Salas, J. M. (1988). Improving the learning rate of back-propagation with the gradient reuse algorithm. Proceedings of the $2^{\text {nd }}$ International conference on neural networks. Vol. 1, pp. 441-447.

Jacobs, R. A. (1988). Increased rates of convergence through learning rate adaptation. Neural Networks, Vol. 1, pp. 295-307.

Khamene, A. and Negahadariapour, S. (2002) A new method for the extraction of fetal ECG from the composite abdominal signal. IEEE Transactions on Biomedical Engineering, Vol. 47, No. , pp. 507-511, April.

Kovaes, E., Torok, M., and Habermajer, I. (2000) A rule-based phonocardiogrphic method for long- term fetal heart rate monitoring. IEEE Transactions on Biomedical Engineering, Vol. 47, pp. 124-130.

Li, C., Zheng, C., and Tai, C. F. (1995). Detection of ECG characteristic points using wavelet transforms. IEEE Transactions on Biomedical Engineering, Vol. 42, January.

Lin, C. et al., (1997) A portable monitor for fetal heart rate and uterine contraction. IEEE Engineering in Medicine and Biology Socierty (EMBS) Magazine, pp. 80-84, Nov./Dec.

Longini, R. L., Reichert, T. A., Yu, J. M. and Crowley, J. S. (1977) Near orthogonal basis functions: A real time fetal ECG technique. IEEE Transactions on Biomedical Engineering, Vol. 24, pp. 39-43.

Moody, G. (1997), MIT/BIH database distribution. MIT, Massachusets Avenue, Room 20A113, Cambridge, MA 02139, USA.

Nikias, C. L. and Petropulu, A. P. (1993). Higher Order Spectral Analysis: A Nonlinear Signal Processing Framework. A. V. Oppenheim Series editor.

Rizk, M., Zgallai, W. A., Carson, E., MacLean, A., \& Grattan, K. (2002) Multi-fractility in Fetal Heart Beat Dynamics. The 2nd European Medical and Biological Engineering Conference, Austria, December.

Rizk M., Zgallai W. A., , McLean, A., Carson, E., and Grattan, K. (2001) Virtues and Vices of Source Separation Using Linear Independent Component Analysis for Blind Source Separation of Non-linearly Coupled and Synchronised Fetal and Mother ECGs. IEEE Engineering in Medicine and Biology Conference, USA.

Rizk, M., Zgallai, W., Morgan, C., ElKhafif, S., Carson, E., \& Grattan, K. (2000) Novel decision strategy for P-wave detection utilising nonlinearly synthesised ECG components and their enhanced pseudospectral resonances. IEE Proceedings Science, Measurement E Technology, Special section on Medical Signal Processing, vol. 147, No. 6, pp. 389-397, November.

Rizk M. and Zgallai, W. (1999) "Higher Order Statistics Are Indispensable Tools in The Analysis of Electrocardiogram Signals," IEE Colloquium on Statistical Signal Processing, January.

Rizk, M, Zgallai, W. A., ElKhafif, S., Carson, E., Grattan, K., Thompson, P. (1999). Highly accurate higher-order statistics based neural network classifier of specific abnormality in electrocardiogram signals. International Conference on Acoustics, Speech, \& Signal Processing, ICASSP99, USA, 13/3.

Rizk, M., Zgallai, W. A., El-Khafif, S., Carson, E., Grattan, K. (1998) “Higher- Order Ambulatory Electrocardiogram Identification and Motion Artifact Suppression With Adaptive Second- and Third-Order Volterra Filters," SPIE '98 Advanced Signal Processing Algorithms, Architectures, and Implementations VIII Vol. 3461 pp. 417-431, San Diego, USA, 19-24 July.

Rizk, M., Romare, D., Zgallai, W. A., Grattan, K., Hardiman, P., and O'Riordan, J. (1995) "Higher order statistics (HOS) in signal processing: Are they of any use?," IEE colloquium, digest \#111, pp. 1/1-1/6, London, May. 
Schetzen, M. (1980) The Volterra Wiener theory of the non- linear systems. New York: Wiley.

Schreiber, T. and Kaplan, D. (1996) Signal separation by nonlinear projections: The fetal Electrocardiogram. Physics Review E, Vol. 53, p. R4326.

Spiegl, A., Steinbigler, Schmueking, P. I., Knez, A. and Haberl, R. (1998). Analysis of beat-tobeat variability of frequency contents in the electrocardiogram using twodimensional Fourier transform. IEEE Transactions on Biomedical Engineering. Vol. 45, No. 2, pp. 235-241, February.

Strube, G. and Strube, G. (1989), Commonsense Cardiology, Kluwer Academic Publishers.

Sureau, C. (1996) Historical perspectives: forgotten past, unpredictable future. Baillier's Clinical obstetrics and gynaecology, international practice and research, intrapartum surveillance, (J Gardosi, ed.), vol. 10, No. 2, pp. 167-184.

Van Bemmel, J. H. (1968) Detection of weak electrocardiograms by autocorrelation and cross correlation envelopes. IEEE Transactions on Biomedical Engineering, Vol. 15, pp. 17-23.

Van Oosterom, A. (1986) Spatial filtering of the fetal electrocardiogram. Journal of Perinatal Medicine, vol. 14, pp. 411-419.

Wallach, E. and Widrow, B. (1984) The Least-Mean Fourth (LMF) adaptive algorithm and its Family. IEEE Transactions on Information Theory, Vol. IT-30, No. 2, pp. 275-283, March.

Widrow, B. et al., (1975) Adaptive Noise Cancellation: Principles and Applications. Proceedings of IEEE, Vol. 63, No. 12, pp. 1692-1716, December.

Zgallai, W. A. (2012) Non-Invasive Fetal Heartbeat Detection Using Bispectral Contour Matching. International Conference on Electronics and Biomedical Engineering Applications, UAE.

Zgallai, W. A. (2011a) A novel super-resolution MUSIC-based pseudo-bispectrum. The IEEE 17th International Conference on Digital Signal Processing (DSP), Greece, 6-8/07.

Zgallai, W. A. (2011b) A polycoherence-based ECG signal non-linear detector. The IEEE 17th International Conference on Digital Signal Processing (DSP), Greece, 6-8/07.

Zgallai, W. A. (2011c) MUSIC pseudo-bispectrum detects ECG ischaemia. The IEEE 17th International Conference on Digital Signal Processing (DSP), Greece, 6-8/07.

Zgallai, W. A. (2010) Non-invasive fetal heartbeat detection using third-order cumulant slices matching and ANN classifiers. The $7^{\text {th }}$ International Association of Science and Technology for Development (IASTED), International Conference on Biomedical Engineering, IASTED, Austria, 17/02.

Zgallai, W. A. (2009) Embedded Volterra for Prediction of Electromyographic Signals During Labour. The 16 $16^{\text {th }}$ IEEE International Conference on Digital Signal Processing (DSP), Greece, 05/07.

Zgallai, W. A. (2007) Advanced Robust Non-Invasive Fetal Heart Detection Techniques During Active Labour Using One Pair of Transabdominal Electrodes, PhD Thesis, City University London, UK.

Zgallai, W. A. Et al., (1997) MUSIC-Based Bispectrum Detector: A Novel Non-Invasive Detection Method For Overlapping Fetal and Maternal ECG Signals. Proc 19th IEEE International Conference Engeering in Medicine and Biology, EMBS, pp. 72-75, USA, October.

Zgallai W. A. et al., (1997) Third-order cumulant signature matching technique for noninvasive fetal heart beat identification. IEEE International Conference on Acoustics, Spech, and Signal Processing (ICASSP), vol. 5, pp 3781-3784, Germany.

M. Sabry-Rizk, S. El-Khafif, E. Carson, W. Zgallai, K. Grattan, C. Morgan and P. Hardiman (1999b), "Suspicious Polyphase Patterns of Normal Looking ECGs Provide Fast Early Diagnoses of a Coronary Artery Disease," IEEE Proceedings of the joint EMBS/BMES conference, pp. 979, 13-16 October 1999. 


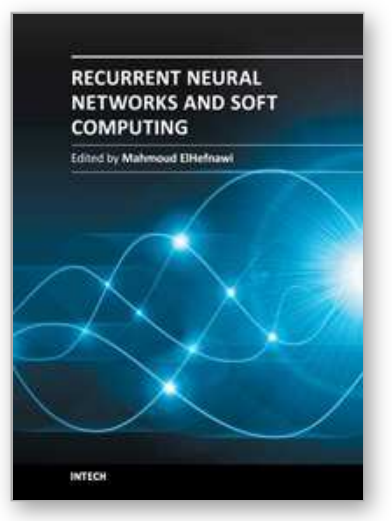

\section{Recurrent Neural Networks and Soft Computing \\ Edited by Dr. Mahmoud ElHefnawi}

ISBN 978-953-51-0409-4

Hard cover, 290 pages

Publisher InTech

Published online 30, March, 2012

Published in print edition March, 2012

\section{How to reference}

In order to correctly reference this scholarly work, feel free to copy and paste the following:

Walid A. Zgallai (2012). Detection and Classification of Adult and Fetal ECG Using Recurrent Neural Networks, Embedded Volterra and Higher-Order Statistics, Recurrent Neural Networks and Soft Computing, Dr. Mahmoud ElHefnawi (Ed.), ISBN: 978-953-51-0409-4, InTech, Available from: http://www.intechopen.com/books/recurrent-neural-networks-and-soft-computing/detection-and-classificationof-biomedical-signals-using-embeded-volterra-and-higher-order-statistic

\section{INTECH}

open science | open minds

\section{InTech Europe}

University Campus STeP Ri

Slavka Krautzeka 83/A

51000 Rijeka, Croatia

Phone: +385 (51) 770447

Fax: +385 (51) 686166

www.intechopen.com

\section{InTech China}

Unit 405, Office Block, Hotel Equatorial Shanghai

No.65, Yan An Road (West), Shanghai, 200040, China

中国上海市延安西路65号上海国际贵都大饭店办公楼405单元

Phone: +86-21-62489820

Fax: +86-21-62489821 
(C) 2012 The Author(s). Licensee IntechOpen. This is an open access article distributed under the terms of the Creative Commons Attribution 3.0 License, which permits unrestricted use, distribution, and reproduction in any medium, provided the original work is properly cited. 